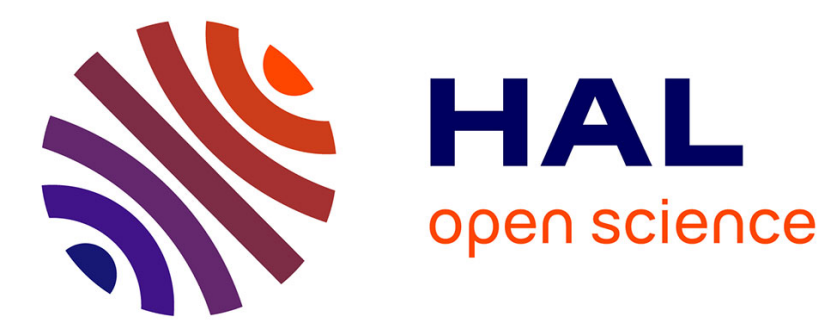

\title{
Probabilistic reachability and control synthesis for stochastic switched systems using the tamed Euler method
}

Adrien Le Coënt, Laurent Fribourg, Jonathan Vacher, Rafael Wisniewski

\section{To cite this version:}

Adrien Le Coënt, Laurent Fribourg, Jonathan Vacher, Rafael Wisniewski. Probabilistic reachability and control synthesis for stochastic switched systems using the tamed Euler method. Nonlinear Analysis: Hybrid Systems, 2020, 36 (100860), 10.1016/j.nahs.2020.100860 . hal-02987885

\section{HAL Id: hal-02987885 \\ https://hal.science/hal-02987885}

Submitted on 4 Nov 2020

HAL is a multi-disciplinary open access archive for the deposit and dissemination of scientific research documents, whether they are published or not. The documents may come from teaching and research institutions in France or abroad, or from public or private research centers.
L'archive ouverte pluridisciplinaire HAL, est destinée au dépôt et à la diffusion de documents scientifiques de niveau recherche, publiés ou non, émanant des établissements d'enseignement et de recherche français ou étrangers, des laboratoires publics ou privés. 


\title{
Probabilistic reachability and control synthesis for stochastic switched systems using the tamed Euler method
}

\author{
Adrien Le Coënt ${ }^{\mathrm{a}}$, Laurent Fribourg ${ }^{\mathrm{b}}$, Jonathan Vacher $^{\mathrm{c}}$, Rafael Wisniewski ${ }^{\mathrm{d}}$ \\ ${ }^{a}$ Department of Computer Science, Aalborg University, \\ Selma Lagerløfs Vej 300, Aalborg, Denmark. \\ ${ }^{b} L S V, C N R S, I N R I A$, ENS Paris-Saclay, \\ 61 Av. du Pdt. Wilson, 94235, Cachan, France. \\ ${ }^{c}$ Albert Einstein College of Medicine, \\ Block Research Pavilion, Department of Systems and Computational Biology, 1301 Morris \\ Park Avenue, 10461 Bronx, NY, USA. \\ ${ }^{d}$ Department of Electronic Systems, Automation and Control, Aalborg University, \\ Fredrik Bajers Vej 7, Aalborg, Denmark.
}

\begin{abstract}
In this paper, we explain how, under the one-sided Lipschitz (OSL) hypothesis, one can find a mean square error bound for a variant of the Euler-Maruyama approximation method for stochastic switched systems. Subsequently, we explain how this bound can be used to control a stochastic switched system in order to make it reach a target zone with guaranteed minimum probability. The method is illustrated on several examples from the literature.
\end{abstract}

Keywords: Stochastic systems, numerical simulation, control system synthesis, switched control systems, nonlinear control systems.

\section{Introduction}

【] Symbolic methods for the verification and control synthesis of hybrid systems (and, particularly, "switched systems") have received significant attention in the past few years. However, control systems involving stochastic differential equations remain difficult to handle with symbolic methods, and few methods have been developed for these systems. One distinguishes two main classes of symbolic methods for hybrid systems: indirect methods and direct methods 2 .

Indirect methods proceed by constructing a finite abstraction of the original system by discretization of the dense state space $\mathbb{R}^{d}$ (where $d$ is the dimension of

This work is supported by the LASSO project financed by an ERC adv. grant; and the DiCyPS project funded by Innovation Fund Denmark.

License CC BY-NC-SA 4.0 : https://creativecommons.org/licenses/by-nc-sa/4.0/

Preprint submitted to Elsevier

January 14, 2020 
the state space). Among the indirect methods, one of the most successful proceeds by approximate bisimulation [10. This method originally designed for deterministic switched systems has been recently extended for stochastic switched systems [30, 31, 32]. This approach relies on the hypothesis of incremental stability of the stochastic switched system (or existence of a common/multiple Lyapunov function). Another method is presented in [7. The associated tool 25] allows to generate formal abstractions for discrete-time Markov processes defined over uncountable (continuous) state spaces.

Indirect methods have also been developed for the verification of safety. Safety verification has been studied using the concept of barrier certificates [23]. The original inquiry has been later modified to cope with stochastic systems [29], and to the design of a controller that makes the closed-loop system safe [28, 24. Also related is the subject of stability and safety verification is the reach avoidance problem. It has been studied using dynamic programming. This approach has been developed both for deterministic hybrid system in [18, and for discrete-time stochastic hybrid systems in 26.

A direct method proceeds by working directly at the level of the dense state of the system $\mathbb{R}^{d}$; it computes "trajectory tubes", which are over-approximations of the set of all the controlled trajectories starting at a given subregion of $\mathbb{R}^{d}$. In previous work, we have followed such a direct approach first in [9], then in [15, using the Euler approximation scheme for calculating over-approximations of tubes of trajectories. We show here how to extend this direct method to stochastic switched systems. The method extends the deterministic approach by replacing the classical Euler approximation scheme with a variant of the stochastic Euler-Maruyama scheme [13. The correctness of these Euler-based methods does not rely on the hypothesis of incremental stability as in [30, 32, but on the hypothesis of 'one-sided Lipschitz (OSL)' condition with constant $\lambda \in \mathbb{R}^{d}$ (also called 'monotonicity'/'dissipativity', see [27]). It can be seen that if a stochastic switched system satisfies an OSL condition with $\lambda<0$, then the function $V\left(x, x^{\prime}\right)=\left\|x-x^{\prime}\right\|^{2}$ is a common incremental Lyapunov function in the sense of [31, from which it follows that the switched system is incrementally stable, and can be analysed by approximate bisimulation. However, Euler-based methods also apply when the system is not incrementally stable, in which case the constant $\lambda$ is necessarily positive. We thus consider a class of systems different from that of 31 .

The plan of the paper is as follows: In Section 3, we give an explicit upper bound on the mean square error of the tamed Euler method for SDEs under OSL condition (Theorem 2). We apply the result in order to ensure reachability properties of stochastic switched systems with guaranteed minimum probability (Section 4). We conclude in Section 5 .

This paper is an extended version of a paper (A. Le Coënt, L. Fribourg and J. Vacher. Control Synthesis for Stochastic Switched Systems using the Tamed Euler Method. In ADHS'18, IFAC-PapersOnLine 51(16), pages 259264. Elsevier Science Publishers, 2018). The main additional material of the extended version is: 
- Section 3.3 , which gives analytical upper bounds on expressions occurring in Theorem 2 ,

- Section 3.4, which gives a lower bound on the probability of reaching a given set $R$ (Proposition 3), and

- Section 4.4, which proposes an optimized method of control synthesis.

\section{Preliminaries}

\subsection{Notations}

The symbols $\mathbb{N}, \mathbb{N}_{\geqslant 0}, \mathbb{R}, \mathbb{R}_{>0}, \mathbb{R}_{\geqslant 0}$ denote the set of natural, nonnegative integer, real, positive, and nonnegative real numbers.

The symbol $\|\cdot\|$ denotes the Euclidean norm on $\mathbb{R}^{d}$. The symbol $\langle\cdot, \cdot\rangle$ denotes the scalar product of two vectors of $\mathbb{R}^{d}$. Given a point $x \in \mathbb{R}^{d}$ and a positive real $r>0$, the ball $B(x, r)$ of centre $x$ and radius $r$ is the set $\left\{y \in \mathbb{R}^{d} \mid\|x-y\| \leqslant r\right\}$.

Throughout this paper, the set $U$ denotes a finite set of switched modes. Given a sequence of $k$ modes $a=\left(a_{1} \cdots a_{k}\right) \in U^{k}$, and a sequence of $p$ modes $b=\left(b_{1} \cdots b_{p}\right) \in \mathbb{N}^{p}$, we denote the concatenation $a * b$ of $a$ and $b$ the sequence of length $k+p: a * b=\left(a_{1} \cdots a_{k} \cdot b_{1} \cdots b_{p}\right)$.

A sequence of non-negative integers is written in bold when it is used as a multi-index, e.g. $\mathbf{a}=\left(a_{1}, \ldots, a_{p}\right) \in \mathbb{N}_{\geqslant 0}^{p}$, the sum of the components of $\mathbf{a}$ is denoted by $|\mathbf{a}|=a_{1}+\cdots+a_{p}$, and $\left(\begin{array}{l}b \\ \mathbf{a}\end{array}\right)=\frac{b !}{a_{1} ! a_{2} ! \ldots a_{p} !}$ is the multinomial coefficient.

\subsection{Systems considered and assumptions}

Let $\tau \in \mathbb{R}_{>0}$ be a fixed real number, let $(\Omega, \mathcal{F}, \mathbb{P})$ be a probability space with normal filtration $\left(\mathcal{F}_{t}\right)_{t \in[0, \tau]}$, let $d, m \in \mathbb{N}$ let $W=\left(W^{(1)}, \ldots, W^{(m)}\right)$ : $[0, \tau] \times \Omega \rightarrow \mathbb{R}^{m}$ be an $m$-dimensional standard $\left(W_{t}\right)_{t \in[0, \tau]}$-Brownian motion and let $x_{0}: \Omega \rightarrow \mathbb{R}^{d}$ be an $\mathcal{F}_{0} / \mathcal{B}\left(\mathbb{R}^{d}\right)$-measurable mapping with $\mathbb{E}\left[\left\|x_{0}\right\|^{p}\right]<\infty$ for all $p \in[1, \infty)$. Moreover, let $f: \mathbb{R}^{d} \rightarrow \mathbb{R}^{d}$ be a continuously differentiable function whose derivative grows at most polynomially. Formally, let us suppose the existence of constants $D \in \mathbb{R}_{\geqslant 0}$ and $q \in \mathbb{N}$ such that, for all $x, y \in \mathbb{R}^{d}$

$$
\|f(x)-f(y)\|^{2} \leqslant D\|x-y\|^{2}\left(1+\|x\|^{q}+\|y\|^{q}\right)
$$

Let $g=\left(g_{i, j}\right)_{i \in\{1, \ldots, d\}, j \in\{1, \ldots, m\}}: \mathbb{R}^{d} \rightarrow \mathbb{R}^{d \times m}$ be a globally Lipschitz continuous function: there exists $L_{g} \in \mathbb{R}_{\geqslant 0}$ such that, for all $x, y \in \mathbb{R}^{d}$

$$
\|g(x)-g(y)\| \leqslant L_{g}\|x-y\|
$$

Finally, let us suppose that $\mathrm{f}$ is globally one-sided Lipschitz with constant $\lambda \in \mathbb{R}$ :

$$
\exists \lambda \in \mathbb{R} \forall x, y \in \mathbb{R}^{d}:\langle f(y)-f(x), y-x\rangle \leqslant \lambda\|y-x\|^{2}
$$

Then consider the Stochastic Differential Equations (SDE):

$$
\mathrm{d} X_{t}=f\left(X_{t}\right) \mathrm{d} t+g\left(X_{t}\right) \mathrm{d} W_{t}, \quad X_{0}=x_{0}
$$


for $t \in[0, \tau]$. The drift coefficient $f$ is the infinitesimal mean of the process $X$ and the diffusion coefficient $g$ is the infinitesimal standard deviation of the process $X$. Under the above assumptions, the SDE (1) is known to have a unique strong solution. More formally, there exists an adapted stochastic process $\left(X_{t, x_{0}}\right):[0, \tau] \times \Omega \rightarrow \mathbb{R}^{d}$ with continuous sample paths fulfilling

$$
X_{t, x_{0}}=x_{0}+\int_{0}^{t} f\left(X_{s}\right) \mathrm{d} s+\int_{0}^{t} g\left(X_{s}\right) \mathrm{d} W_{s}
$$

for all $t \in[0, \tau] \mathbb{P}$-a.s. (see, e.g., $([21])$ ).

We denote by $X_{t, x_{0}}$ the solution of Equation (1) at time $t$ from initial condition $X_{0, x_{0}}=x_{0} \mathbb{P}$-a.s., in which $x_{0}$ is a random variable that is measurable in $\mathcal{F}_{0}$.

Remark 1. Constants $\lambda, L_{g}$ and $D$ can be computed using (constrained) optimization algorithms (see ([15])).

\subsection{Overview of the approach}

We aim at synthesizing reachability controllers for the class of systems described above. Our approach consists in adapting reachability analysis based control systhesis methods to stochastic systems. In [15, we successfully used the Euler method for guaranteeing controlled reachability of deterministic systems using the Euler method in association to a new error bound relying on the OSL property of the vector field.

Consider equation (1) with $g=0$. Classically, one knows that, if the function $f$ is Lipschitz continuous with Lipschitz constant $L$, the solution of the ODE starting at a given initial value exists and is unique. Besides, one has:

$$
\left\|X_{t, x_{0}}-X_{t, x_{1}}\right\| \leqslant e^{L t}\left\|x_{0}-x_{1}\right\|,
$$

with two initial values $x_{i}(i=0,1)$. This gives a rough growth bound, i.e. a function bounding the distance of neighboring trajectories as $t$ evolves. In [6], it is proven that, if $f$ is continuous and OSL with OSL constant $\lambda$, then the solution of the ODE starting at a given initial value exists and is unique, and, for all $x_{0}, x_{1} \in \mathbb{R}^{n}$ :

$$
\left\|X_{t, x_{0}}-X_{t, x_{1}}\right\| \leqslant e^{\lambda t}\left\|x_{0}-x_{1}\right\| .
$$

This gives a more accurate growth bound because a Lipschitz function $f$ is always OSL, and the associated OSL constant $\lambda$ is always less than or equal to its Lipschitz counterpart $L$. Using the OSL constant $\lambda$, it is also possible to bound the error $\left\|X_{t, x_{0}}-\tilde{X}_{t, x_{1}}\right\|$ in function of $\left\|x_{0}-x_{1}\right\|$, where $\tilde{X}_{t, x_{1}}$ denotes the Euler approximate trajectory of the solution $X_{t, x_{1}}$. We can then conclude that any trajectory starting from $x_{0}$ close enough from a given initial condition $x_{1}\left(\left\|x_{0}-x_{1}\right\| \leqslant \delta_{0}\right)$ will remain within some bound (denoted by $\delta_{t, \delta_{0}}$ ) of the Euler trajectory $\tilde{X}_{t, x_{1}}$. Our objective is to use a similar approach for stochastic systems, while making as few additional hypotheses on the dynamics as possible. 
Therefore, we first explain how we can use a variant of the Euler-Maruyama scheme called tamed Euler scheme in order to guarantee reachability of the expectation of the state $\mathbb{E}\left[X_{t, x_{0}}\right]$ of the stochastic system (1) in a given set. It consists in computing an approximate Euler-based trajectory $\tilde{X}_{t, x_{1}}$, we then provide an error bound for $\mathbb{E}\left[\left\|X_{t, x_{0}}-\tilde{X}_{t, x_{1}}\right\|^{2}\right]$ (Theorem 22) which allows to write a result for the expectation of the non approximated trajectory $\mathbb{E}\left[X_{t, x_{0}}\right]$ (Proposition 2). This result can be extended further as a probability result (the probability with which the state reaches the set, Proposition 3).

This reachability analysis method is then used in association with tiling based control synthesis algorithms to ensure that the system is stabilised in a given region of interest with a given probability (Section 4). The first algorithm we use is based on an adaptive state-space decomposition algorithm [15, 14], and uses an exhaustive enumeration of all possible control sequences (up to a given length) in order to find stabilising sequences (see Section 4.3). We then propose a faster approach (in Section 4.4) which relies on state-space discretization and dynamic programming techniques in order to minimize the average distance of the state with some given objective after a given number of steps. The second algorithm is merely an enhancement of the control sequence search of the first one, using a static tiling. Note that with both algorithms, there is no need to compute a finite-state abstraction of the system, which means that computing a controller is done with the application of a single algorithm, unlike [31] which requires the computation of a finite-state abstraction before computing controllers.

Example 1. Throughout the paper, we illustrate our approach on a 2-mode switched system borrowed from [30]. This case-study is a stochastic version of a well-known system originally introduced as an illustrative example in [16]. The dynamics is given by the system of equations

$\mathrm{d} x_{1}=\left(-0.25 x_{1}+p x_{2}+(-1)^{p} 0.25\right) \mathrm{d} t+w x_{1} \mathrm{~d} W_{t}^{1}$,

$\mathrm{d} x_{2}=\left((p-3) x_{1}-0.25 x_{2}+(-1)^{p}(3-p)\right) \mathrm{d} t+w x_{2} \mathrm{~d} W_{t}^{2}$,

where $p=1,2$ is the mode, and we consider different values of noise $w \in \mathbb{R}_{>0}$.

\section{Bounding the Error of the tamed Euler method}

\subsection{Tamed Euler approximation}

The standard way to extend the classical Euler method for ordinary differential equations to the SDE (1) is the Euler-Maruyama scheme ([19]). More precisely, given $z: \Omega \rightarrow \mathbb{R}^{d}$ an $\mathcal{F}_{0} / \mathcal{B}\left(\mathbb{R}^{d}\right)$-measurable mapping with $\mathbb{E}\left[\|z\|^{p}\right]<\infty$ for all $p \in[1, \infty)$, the explicit Euler-Maruyama (EM) method for the SDE (1) is given by the mappings $Y_{n, z}^{N}: \Omega \rightarrow \mathbb{R}^{d}, n \in\{0,1, \ldots, N\}$, which satisfy $Y_{0, z}^{N}=z$ and

$$
Y_{n+1, z}^{N}=Y_{n, z}^{N}+\frac{\tau}{N} \cdot f\left(Y_{n, z}^{N}\right)+g\left(Y_{n, z}^{N}\right)\left(W_{(n+1) \tau / N}-W_{n \tau / N}\right)
$$

for all $n \in\{0,1, \ldots, N-1\}$ and all $N \in \mathbb{N}$. See ([19]). Unfortunately, the convergence results for the EM scheme does not hold when the drift function 
$f$ of the SDE (1) is polynomial (not an affine function). In order to consider non affine drift functions, we adopt a refined scheme, which has been proposed recently to overcome this difficulty ([13]). Let $\underline{X}_{n, z}^{N}: \Omega \rightarrow \mathbb{R}^{d}$,

$$
\begin{aligned}
\underline{X}_{n+1, z}^{N}= & \underline{X}_{n, z}^{N}+\frac{\frac{\tau}{N} \cdot f\left(\underline{X}_{n, z}^{N}\right)}{1+\frac{\tau}{N} \cdot\left\|f\left(\underline{X}_{n, z}^{N}\right)\right\|} \\
& +g\left(\underline{X}_{n, z}^{N}\right)\left(W_{\frac{(n+1) \tau}{N}}-W_{\frac{n \tau}{N}}\right)
\end{aligned}
$$

for all $n \in\{0,1, \ldots, N-1\}$ and all $N \in \mathbb{N}$. We refer to the numerical method (4) as a tamed Euler scheme ([13] $)$. In this method the drift term $\frac{\tau}{n} \cdot f\left(\underline{X}_{n, z}^{N}\right)$ is "tamed" by the factor $1 /\left(1+\frac{\tau}{N} \cdot\left\|f\left(\underline{X}_{n, z}^{N}\right)\right\|\right)$ for $n \in\{0,1, \ldots, N-1\}$ and $N \in \mathbb{N}$ in (4). In order to establish results for the time continuous system, we use the following time continuous interpolation of the time discrete numerical approximations [4], also introduced in ([13]). Let $\tilde{X}_{z}^{N}:[0, \tau] \times \Omega \rightarrow \mathbb{R}^{d}, N \in \mathbb{N}$, be a sequence of stochastic processes given by

$$
\tilde{X}_{t, z}^{N}=\tilde{X}_{n, z}^{N}+\frac{(t-n \tau / N) \cdot f\left(\tilde{X}_{n, z}^{N}\right)}{1+\tau / N \cdot\left\|f\left(\tilde{X}_{n, z}^{N}\right)\right\|}+g\left(\tilde{X}_{n, z}^{N}\right)\left(W_{t}-W \frac{n \tau}{N}\right)
$$

for all $t \in\left[\frac{n \tau}{N}, \frac{(n+1) \tau}{N}\right], n \in\{0,1 \ldots, N-1\}$ and all $N \in \mathbb{N}$. Note that $\tilde{X}_{t, z}^{N}$ : $[0, \tau] \times \Omega \rightarrow \mathbb{R}^{d}$ is an adapted stochastic process with continuous sample paths for every $N \in \mathbb{N}$.

We finally introduce a piecewise constant interpolation $\underline{X}_{t, z}^{N}$ of the tamed Euler scheme as follows. It is mainly used in some constants when establishing the error bound and for proving Theorem 2 .

$$
\underline{X}_{t, z}^{N}:=\underline{X}_{n, z}^{N} \quad \text { for } t \in\left[\frac{n \tau}{N}, \frac{(n+1) \tau}{N}\right) .
$$

Note that $\tilde{X}_{t, z}^{N}=\underline{X}_{t, z}^{N}=\underline{X}_{n, z}^{N}$ at time $t=\frac{n \tau}{N}$ for $n \in\{0,1, \ldots, N\}$.

The following theorem is proven in ([13]):

Theorem 1. Let us suppose $(\overline{\mathrm{H} 1})$ - $(\mathrm{H} 2)-(\sqrt[\mathrm{H} 3]{2})$. Let $z: \Omega \rightarrow \mathbb{R}^{d}$ be an $\mathcal{F}_{0} / \mathcal{B}\left(\mathbb{R}^{d}\right)$ measurable mapping with $\mathbb{E}\left[\|z\|^{p}\right]<\infty$ for all $p \in[1, \infty)$. Then, for all $p \in[1, \infty)$, the tamed Euler scheme (4) satisfies:

$$
\sup _{N \in \mathbb{N}} \sup _{n \in\{0,1, \ldots, N\}} \mathbb{E}\left[\left\|\underline{X}_{n, z}^{N}\right\|^{p}\right]<\infty
$$

This theorem allows to ensure the strong convergence of the tamed Euler method. Any number $N$ of subsampling steps can thus be used. This number is now left implicit for the sake of simplicity. From Theorem 1 it follows (cf. Lemma 4.3, ([11])):

Lemma 1. Let us suppose $(\overline{\mathrm{H} 1} 1)-(\overline{\mathrm{H} 2})-(\overline{\mathrm{H} 3})$. Let $z: \Omega \rightarrow \mathbb{R}^{d}$ be an $\mathcal{F}_{0} / \mathcal{B}\left(\mathbb{R}^{d}\right)$ measurable mapping with $\mathbb{E}\left[\|z\|^{p}\right]<\infty$ for all $p \in[1, \infty)$. Let us consider the time continuous interpolation of the tamed Euler scheme as defined in (5), and 
the piecewise constant interpolation as defined in (6). Then, for any even integer $r \geqslant 2$, there exist two constants $E_{r, z}$ and $F_{r, z}$ such that

$$
\sup _{0 \leqslant t \leqslant \tau} \mathbb{E}\left\|\underline{X}_{t, z}-\tilde{X}_{t, z}\right\|^{r} \leqslant\left(\Delta_{t}\right)^{\frac{r}{2}}\left(E_{r, z}\left(\Delta_{t}\right)^{\frac{r}{2}}+F_{r, z} d\right) .
$$

with $\Delta_{t}=\tau / N$ and:

$$
\begin{aligned}
& E_{r, z}=2^{r}\left(\|f(0)\|^{r}+D 2^{\frac{r+1}{2}}\right. \\
& \left.\qquad\left(1+\mathbb{E} \sup _{0 \leqslant t \leqslant \tau}\left\|\underline{X}_{t, z}\right\| \|^{q r}\right)^{\frac{1}{2}}\left(\mathbb{E} \sup _{0 \leqslant t \leqslant \tau}\left\|\underline{X}_{t, z}\right\|^{2 r}\right)^{\frac{1}{2}}\right), \\
& F_{r, z}=2^{r}\left(\|g(0)\|^{2 r}+L_{g}^{r} \mathbb{E} \sup _{0 \leqslant t \leqslant \tau}\left\|\underline{X}_{t, z}\right\|^{\frac{r}{2}}\right) .
\end{aligned}
$$

Remark 2. Constants $E_{r, z}$ and $F_{r, z}$ are computed using the constants $\lambda$ and $L_{g}$ (see Remark 1), and the expected values of $\sup _{0 \leqslant t \leqslant \tau}\left\|\underline{X}_{t, z}\right\|^{p}$ for the required values of $p$ at each time $t=0, \Delta t, 2 \Delta t, \ldots, N \Delta t$. These expected values can either be computed numerically by using a Monte Carlo method, or by evaluating analytically the expectations $\mathbb{E} \sup _{0 \leqslant t \leqslant \tau}\left\|\underline{X}_{t, z}\right\|^{p}$ for all required values of $p$. Proposition 2 explains how such expectations can be computed for the piecewise linear interpolation $\tilde{X}_{t, z}$, the computation for $\underline{X}_{t, z}^{p}$ is the same but much simpler since there is no time dependent term in (6). In this paper, we use the latter approach.

\subsection{Mean square error bounding}

The following Theorem holds for SDE (1). This corresponds to a stochastic version of Theorem 1 of ([15]), showing that a similar result holds on average, using the tamed Euler method of ([13]). It is an adaptation of Theorem 4.4 in $([1])$.

Theorem 2. Given the SDE system (1) satisfying $\sqrt{\mathrm{H} 1}-(\sqrt{\mathrm{H} 2})-(\mathrm{H} 3)$. Let $\delta_{0} \in$ $\mathbb{R}_{\geqslant 0}$. Suppose that $z$ is a random variable on $\mathbb{R}^{d}$ such that

$$
\mathbb{E}\left[\left\|x_{0}-z\right\|^{2}\right] \leqslant \delta_{0}^{2} .
$$

Then, we have, for all $\tau \geqslant 0$ :

$$
\mathbb{E}\left[\sup _{0 \leqslant t \leqslant \tau}\left\|X_{t, x_{0}}-\tilde{X}_{t, z}\right\|^{2}\right] \leqslant \delta_{\tau, \delta_{0}}^{2},
$$

with $\delta_{\tau, \delta_{0}}^{2}:=\beta(\tau) e^{\gamma \tau}$, where:

$$
\begin{aligned}
& \gamma=2\left(\sqrt{\Delta_{t}}+2 \lambda+L_{g}^{2}+128 L_{g}^{4}\right), \text { and } \\
& \qquad \begin{array}{l}
\beta(\tau)=2 \delta_{0}^{2}+2 \tau \Delta_{t} L_{g}^{2}\left(1+128 L_{g}^{2}\right)\left(F_{2, z} d+E_{2, z} \Delta_{t}\right) \\
+4 \tau \sqrt{\Delta_{t}} D\left(F_{4, z} d+E_{4, z} \Delta_{t}^{2}\right)^{\frac{1}{2}} \\
\left(1+4 \mathbb{E} \sup _{0 \leqslant t \leqslant \tau}\left\|\underline{X}_{t, z}\right\|^{2 q}+4 \mathbb{E} \sup _{0 \leqslant t \leqslant \tau}\left\|\tilde{X}_{t, z}\right\|^{2 q}\right)^{\frac{1}{2}} .
\end{array}
\end{aligned}
$$

with $\Delta_{t}=\tau / N$. 
Proof. The proof closely follows the proof of Theorem 4.4 in ([1]). Let $e_{t}=$ $X_{t, x_{0}}-\tilde{X}_{t, z}$. We have, for all $0 \leqslant t \leqslant \tau$ :

$$
\mathrm{d} e_{t}=\left(f\left(X_{t, x_{0}}\right)-f(z)\right) \mathrm{d} t+\left(g\left(X_{t, x_{0}}\right)-g(z)\right) \mathrm{d} W_{t} .
$$

Then, by using Equation (8) and the integral version of Itô formula applied to function $x \mapsto\|x\|^{2}$ we obtain

$$
\begin{aligned}
& \left\|e_{t}\right\|^{2}=\left\|e_{0}\right\|^{2}+\int_{0}^{t} 2\left\langle e_{s}, f\left(X_{s, x_{0}}\right)-f\left(\underline{X}_{s, z}\right)\right\rangle \mathrm{d} s \\
& +\int_{0}^{t}\left\|g\left(X_{s, x_{0}}\right)-g\left(\underline{X}_{s, z}\right)\right\|^{2} \mathrm{~d} s+M(t),
\end{aligned}
$$

where $e_{0}=x_{0}-z$, and

$$
M(t)=\int_{0}^{t} 2\left\langle e_{s}, g\left(X_{s, x_{0}}\right)-g\left(\underline{X}_{s, z}\right)\right\rangle \mathrm{d} W_{s} .
$$

So we have using $(\mathrm{H} 2)$ :

$$
\begin{aligned}
& \left\|e_{t}\right\|^{2} \leqslant\left\|e_{0}\right\|^{2}+\int_{0}^{t} 2\left\langle e_{s}, f\left(X_{s, x_{0}}\right)-f\left(\tilde{X}_{s, z}\right)\right\rangle \mathrm{d} s \\
& +L_{g}^{2} \int_{0}^{t}\left\|X_{s, x_{0}}-\underline{X}_{s, z}\right\|^{2} \mathrm{~d} s \\
& +\int_{0}^{t} 2\left\langle e_{s}, f\left(\tilde{X}_{s, z}\right)-f\left(\underline{X}_{s, z}\right)\right\rangle \mathrm{d} s+M(t) .
\end{aligned}
$$

So we have using $(\mathrm{H} 3)$ and Young's inequality:

$$
\begin{aligned}
& \left\|e_{t}\right\|^{2} \leqslant\left\|e_{0}\right\|^{2}+\int_{0}^{t}\left(2 \lambda\left\|e_{s}\right\|^{2}+L_{g}^{2}\left\|e_{s}\right\|^{2}\right) \mathrm{d} s \\
& +L_{g}^{2} \int_{0}^{t}\left\|\underline{X}_{s, z}-\tilde{X}_{s, z}\right\|^{2} \mathrm{~d} s \\
& +\int_{0}^{t}\left(\frac{1}{\sqrt{\Delta_{t}}}\left\|f\left(\tilde{X}_{s, z}\right)-f\left(\underline{X}_{s, z}\right)\right\|^{2}+\sqrt{\Delta_{t}}\left\|e_{s}\right\|^{2}\right) \mathrm{d} s+M(t) .
\end{aligned}
$$

So we have using ( $1 \mathrm{H} 1)$, for all $0 \leqslant t \leqslant \tau$ :

$$
\begin{aligned}
& \left\|e_{t}\right\|^{2} \leqslant\left\|e_{0}\right\|^{2}+\left(\sqrt{\Delta_{t}}+2 \lambda+L_{g}^{2}\right) \int_{0}^{t}\left\|e_{s}\right\|^{2} \mathrm{~d} s \\
& +L_{g}^{2} \int_{0}^{t}\left\|\underline{X}_{s, z}-\tilde{X}_{s, z}\right\|^{2} \mathrm{~d} s \\
& +\frac{D}{\sqrt{\Delta_{t}}} \int_{0}^{t}\left(1+\left\|\underline{X}_{s, z}\right\|^{q}+\left\|\tilde{X}_{s, z}\right\|^{q}\right)\left\|\underline{X}_{s, z}-\tilde{X}_{s, z}\right\|^{2} \mathrm{~d} s \\
& +M(t) .
\end{aligned}
$$


It follows using Lemma 1 for $r=2$, and Cauchy-Schwarz inequality:

$$
\begin{aligned}
& \mathbb{E}\left[\sup _{0 \leqslant s \leqslant t}\left\|e_{s}\right\|^{2}\right] \leqslant \mathbb{E}\left\|e_{0}\right\|^{2}+\left(\sqrt{\Delta_{t}}+2 \lambda+L_{g}^{2}\right) \int_{0}^{t} \mathbb{E}\left\|e_{s}\right\|^{2} \mathrm{~d} s \\
& +L_{g}^{2} \tau \Delta_{t}\left(E_{2, z} \Delta_{t}+F_{2, z} d\right) \\
& +\frac{D}{\sqrt{\Delta_{t}}} \\
& \int_{0}^{t}\left(\mathbb{E}\left(1+\left\|\underline{X}_{s, z}\right\|^{q}+\left\|\tilde{X}_{s, z}\right\|^{q}\right)^{2}\right)^{\frac{1}{2}}\left(\mathbb{E}\left\|\underline{X}_{s, z}-\tilde{X}_{s, z}\right\|^{4}\right)^{\frac{1}{2}} \mathrm{~d} s \\
& +m(t),
\end{aligned}
$$

where

$$
m(t)=\mathbb{E}\left[\sup _{0 \leqslant s \leqslant t}\|M(s)\|\right] .
$$

Hence, using using Lemma 1 for $r=4$, and inequality $(a+b)^{p} \leqslant 2^{p}\left(a^{p}+b^{p}\right)$ :

$$
\begin{aligned}
& \left.\mathbb{E}\left[\sup _{0 \leqslant s \leqslant t}\left\|e_{s}\right\|^{2}\right] \leqslant \mathbb{E}\left\|e_{0}\right\|^{2}+\left(\sqrt{\Delta_{t}}+2 \lambda+L_{g}^{2}\right)\right) \int_{0}^{t} \mathbb{E}\left\|e_{s}\right\|^{2} \mathrm{~d} s \\
& +L_{g}^{2} \tau \Delta_{t}\left(E_{2, z} \Delta_{t}+F_{2, z} d\right) \\
& +2 D \tau \sqrt{\Delta_{t}}\left(E_{4, z} \Delta_{t}^{2}+F_{4, z} d\right)^{\frac{1}{2}} \\
& \left(1+4 \mathbb{E} \sup _{0 \leqslant t \leqslant \tau}\left\|\underline{X}_{t, z}\right\|^{2 q}+4 \mathbb{E} \sup _{0 \leqslant t \leqslant \tau}\left\|\tilde{X}_{t, z}\right\|^{2 q}\right)^{\frac{1}{2}}+m(t) .
\end{aligned}
$$

On the other hand, from the Burkholder-Davis-Gundy inequality, we get:

$$
m(t) \leqslant 16 \mathbb{E}\left[\int_{0}^{t}\left\|e_{s}\right\|^{2}\left\|g\left(X_{s, x_{0}}\right)-g\left(\underline{X}_{s, z}\right)\right\|^{2} \mathrm{~d} s\right]^{\frac{1}{2}}
$$

Hence, using (H2):

$$
m(t) \leqslant 16 L_{g}^{2} \mathbb{E}\left[\sup _{0 \leqslant s \leqslant t}\left\|e_{s}\right\|^{2} \int_{0}^{t}\left\|X_{s, x_{0}}-\underline{X}_{s, z}\right\|^{2} \mathrm{~d} s\right]^{\frac{1}{2}}
$$

Then, using Young's inequality (for any $\alpha>0$ ):

$$
m(t) \leqslant 8 L_{g}^{2}\left(\alpha \mathbb{E}\left[\sup _{0 \leqslant s \leqslant t}\left\|e_{s}\right\|^{2}\right]+\frac{1}{\alpha} \mathbb{E}\left[\int_{0}^{t}\left\|X_{s, x_{0}}-\underline{X}_{s, z}\right\|^{2} \mathrm{~d} s\right]\right) .
$$

Hence, by using Lemma 1 for $r=2$ :

$$
\begin{aligned}
& m(t) \leqslant 8 \alpha L_{g}^{2} \mathbb{E}\left[\sup _{0 \leqslant s \leqslant t}\left\|e_{s}\right\|^{2}\right] \\
& +\frac{8 L_{g}^{2}}{\alpha} \int_{0}^{t} \mathbb{E}\left[\sup _{0 \leqslant r \leqslant s}\left\|e_{r}\right\|^{2}\right] \mathrm{d} s \\
& +\frac{8 L_{g}^{2}}{\alpha} \tau \Delta_{t}\left(E_{2, z} \Delta_{t}+F_{2, z} d\right) .
\end{aligned}
$$


Hence, letting $\alpha=\frac{1}{16 L_{g}^{2}}$, we have by replacing in 14 :

$$
\begin{aligned}
& \frac{1}{2} \mathbb{E}\left[\sup _{0 \leqslant s \leqslant t}\left\|e_{s}\right\|^{2}\right] \leqslant \\
& \delta_{0}^{2}+\left(\sqrt{\Delta_{t}}+2 \lambda+L_{g}^{2}+128 L_{g}^{4}\right) \int_{0}^{t} \mathbb{E}\left[\sup _{0 \leqslant r \leqslant s}\left\|e_{r}\right\|^{2}\right] \mathrm{d} s \\
& +\tau\left(L_{g}^{2}+128 L_{g}^{4}\right) \Delta_{t}\left(E_{2, z} \Delta_{t}+F_{2, z} d\right) \\
& +\tau 2 D \sqrt{\Delta_{t}}\left(E_{4, z} \Delta_{t}^{2}+F_{4, z} d\right)^{\frac{1}{2}} \\
& \left(1+4 \mathbb{E} \sup _{0 \leqslant t \leqslant \tau}\left\|\underline{X}_{t, z}\right\|^{2 q}+4 \mathbb{E} \sup _{0 \leqslant t \leqslant \tau}\left\|\tilde{X}_{t, z}\right\|^{2 q}\right)^{\frac{1}{2}} .
\end{aligned}
$$

It results from Gronwall's inequality:

$$
\mathbb{E}\left[\sup _{0 \leqslant t \leqslant \tau}\left\|e_{t}\right\|^{2}\right]=\beta(\tau) e^{\gamma \tau},
$$

with

$$
\begin{aligned}
& \gamma=2\left(\sqrt{\Delta_{t}}+2 \lambda+L_{g}^{2}+128 L_{g}^{4}\right), \text { and } \\
& \quad \beta(\tau)=2 \delta_{0}^{2}+2 \tau\left(\Delta_{t} L_{g}^{2}\left(1+128 L_{g}^{2}\right)\left(F_{2, z} d+E_{2, z} \Delta_{t}\right)\right. \\
& +4 \tau \sqrt{\Delta_{t}} D\left(F_{4, z} d+E_{4, z} \Delta_{t}^{2}\right)^{\frac{1}{2}} \\
& \left(1+4 \mathbb{E} \sup _{0 \leqslant t \leqslant \tau}\left\|\underline{X}_{t, z}\right\|^{2 q}+4 \mathbb{E} \sup _{0 \leqslant t \leqslant \tau}\left\|\tilde{X}_{t, z}\right\|^{2 q}\right)^{\frac{1}{2}} .
\end{aligned}
$$

It follows from Theorem 2 and Jensen's inequality:

Proposition 1. Consider two points $x_{0}$ and $z$ in $\mathbb{R}^{d}$, and a positive real number $\delta_{0}$. Suppose that $x_{0} \in B\left(z, \delta_{0}\right)$. Then $\mathbb{E} X_{t, x_{0}} \in B\left(\tilde{X}_{t, z}, \delta_{t, \delta_{0}}\right)$ for all $t \in[0, \tau]$, where $\delta_{t, \delta_{0}}$ is defined in (7).

\subsection{Numerical pre-computation of expectations}

Theorem 2 requires the knowledge of $\mathbb{E} \sup _{0 \leqslant t \leqslant \tau}\left\|\tilde{X}_{t, z}\right\|^{2 q}$. In this section, we give a pre-computable upper bound of this quantity.

Proposition 2. Let $\tilde{X}_{t, z}$ be the piecewise linear interpolation of the solution to equation (1) as defined in equation (5), written in the form $\tilde{X}_{t, z}=\alpha(z)+$ $\beta(z) t+\gamma(z) W_{t}$ with $\alpha(z)=\tilde{X}_{n, z}^{N}-\frac{(n \tau / N) \cdot f\left(\tilde{X}_{n, z}^{N}\right)}{1+\tau / N \cdot\left\|f\left(\tilde{X}_{n, z}^{N}\right)\right\|}, \beta(z)=\frac{f\left(\tilde{X}_{n, z}^{N}\right)}{1+\tau / N \cdot\left\|f\left(\tilde{X}_{n, z}^{N}\right)\right\|}$, $\gamma(z)=g\left(\tilde{X}_{n, z}^{N}\right)$. Then

$$
\mathbb{E}\left[\left\|\tilde{X}_{t, z}\right\|^{2 q}\right]=\sum_{|\mathbf{k}|=q}\left(\begin{array}{l}
q \\
\mathbf{k}
\end{array}\right) C_{k_{1}, k_{2}, k_{3}}(z) \mu_{k_{4}, k_{5}, k_{6}}(z, 1) t^{k_{2}+2 k_{3}+k_{4}+\frac{k_{4}+k_{5}}{2}+k_{6}},
$$


where $\mathbf{k}=\left(k_{1}, \ldots, k_{6}\right)$ and

$$
C_{k_{1}, k_{2}, k_{3}}(z)=\left(\alpha(z)^{T} \alpha(z)\right)^{k_{1}}\left(2 \alpha(z)^{T} \beta(z)\right)^{k_{2}}\left(\beta(z)^{T} \beta(z)\right)^{k_{3}}
$$

and

$$
\mu_{\kappa}(z, t)=\left\{\begin{array}{c}
\lambda_{\kappa}^{(1)} D_{\frac{k_{5}-k_{4}}{2}, k_{4}, k_{6}}\left(b(z) b(z)^{T}, P_{a, b}(z), Q(z)\right) t^{\frac{k_{4}+k_{5}}{2}+k_{6}} \\
\text { if } k_{4}<k_{5} \quad \text { and } k_{4}+k_{5} \equiv 0(\bmod 2) \\
\lambda_{\kappa}^{(2)} D_{\frac{k_{4}-k_{5}}{2}, k_{5}, k_{6}}\left(a(z) a(z)^{T}, P_{a, b}(z), Q(z)\right) t^{\frac{k_{4}+k_{5}}{2}+k_{6}} \\
\text { if } k_{4}>k_{5} \quad \text { and } k_{4}+k_{5} \equiv 0(\bmod 2) \\
\lambda_{\kappa}^{(3)} D_{k_{5}, k_{6}}\left(P_{a, b}(z), Q(z)\right) t^{k_{5}+k_{6}} \\
\text { if } k_{4}=k_{5} \text { and } k_{5} \equiv 0(\bmod 2) \\
0 \text { if } k_{4}+k_{5} \equiv 1(\bmod 2)
\end{array}\right.
$$

where $a(z)=2 \beta(z)^{T} \gamma(z), b(z)=2 \alpha(z)^{T} \gamma(z), Q(z)=\gamma(z)^{T} \gamma(z)$ and $D_{n_{1}, \ldots, n_{i}}\left(A_{1}, \ldots, A_{i}\right)$ being the normalized Davis polynomials [5, 12] and

$$
\begin{aligned}
P_{a, b}(z) & =\frac{a(z) b(z)^{T}+b(z) a(z)^{T}}{2}, \\
\lambda_{\kappa}^{(1)} & =2^{\frac{k_{4}+k_{5}}{2}+k_{6}-1} k_{4} k_{6}\left(k_{5}-k_{4}\right), \\
\lambda_{\kappa}^{(2)} & =2^{\frac{k_{4}+k_{5}}{2}+k_{6}-1} k_{5} k_{6}\left(k_{4}-k_{5}\right), \\
\lambda_{\kappa}^{(3)} & =2^{k_{5}+k_{6}} k_{5} k_{6} .
\end{aligned}
$$

Proof. First, note that we can write $\tilde{X}_{t, z}$ as the sum of a mean value, a linear drift and a standard Wiener process

$$
\tilde{X}_{t, z}=\alpha(z)+\beta(z) t+\gamma(z) W_{t}
$$

for $z \in \mathbb{R}^{d}$. Then, the squared norm of $\tilde{X}_{t, z}$ is

$$
\begin{aligned}
\tilde{X}_{t, z}^{T} \tilde{X}_{t, z}= & \left(\alpha(z)+\beta(z) t+\gamma(z) W_{t}\right)^{T}\left(\alpha(z)+\beta(z) t+\gamma(z) W_{t}\right) \\
= & \alpha(z)^{T} \alpha(z)+2\left(\alpha(z)^{T} \beta(z)+\beta(z)^{T} \gamma(z) W_{t}\right) t \\
& +\beta(z)^{T} \beta(z) t^{2}+2 \alpha(z)^{T} \gamma(z) W_{t}+W_{t}^{T} \gamma(z)^{T} \gamma(z) W_{t} .
\end{aligned}
$$

The Newton multinomial formula allows to express the $2 q^{\text {th }}$ power of the $\tilde{X}_{t, z}$ norm

$$
\begin{aligned}
\left\|\tilde{X}_{t, z}\right\|^{2 q}=\sum_{|\mathbf{k}|=q}\left(\begin{array}{c}
q \\
\mathbf{k}
\end{array}\right)\left(\alpha(z)^{T} \alpha(z)\right)^{k_{1}}\left(2 \alpha(z)^{T} \beta(z) t\right)^{k_{2}}\left(\beta(z)^{T} \beta(z) t^{2}\right)^{k_{3}} \\
\left(2 \beta(z)^{T} \gamma(z) W_{t} t\right)^{k_{4}}\left(2 \alpha(z)^{T} \gamma(z) W_{t}\right)^{k_{5}}\left(W_{t}^{T} \gamma(z)^{T} \gamma(z) W_{t}\right)^{k_{6}}
\end{aligned}
$$


where $\mathbf{k}=\left(k_{1}, \ldots, k_{6}\right)$. The previous equation can be written as follows

$$
\left\|\tilde{X}_{t, z}\right\|^{2 q}=\sum_{|\mathbf{k}|=q}\left(\begin{array}{l}
q \\
\mathbf{k}
\end{array}\right) C_{k_{1}, k_{2}, k_{3}}(z) t^{k_{2}+2 k_{3}+k_{4}}\left(a(z)^{T} W_{t}\right)^{k_{4}}\left(b(z)^{T} W_{t}\right)^{k_{5}}\left(W_{t}^{T} Q(z) W_{t}\right)^{k_{6}}
$$

where $a(z)=2 \beta(z)^{T} \gamma(z), b(z)=2 \alpha(z)^{T} \gamma(z), Q(z)=\gamma(z)^{T} \gamma(z)$ and

$$
C_{k_{1}, k_{2}, k_{3}}(z)=\left(\alpha(z)^{T} \alpha(z)\right)^{k_{1}}\left(2 \alpha(z)^{T} \beta(z)\right)^{k_{2}}\left(\beta(z)^{T} \beta(z)\right)^{k_{3}} .
$$

We denote

$$
\mu_{\kappa}(z, t) \stackrel{\text { def. }}{=} \mathbb{E}\left[\left(a(z)^{T} W_{t}\right)^{k_{4}}\left(b(z)^{T} W_{t}\right)^{k_{5}}\left(W_{t}^{T} Q(z) W_{t}\right)^{k_{6}}\right]
$$

where $\boldsymbol{\kappa}=\left(k_{4}, k_{5}, k_{6}\right)$. The expectation $\mu_{\kappa}(z, t)$ can always be written as the high-order moment of a product of three quadratic forms [17, therefore we have

$$
\mu_{\kappa}(z, t)=\left\{\begin{array}{c}
\lambda_{\kappa}^{(1)} D_{\frac{k_{5}-k_{4}}{2}, k_{4}, k_{6}}\left(b(z) b(z)^{T}, P_{a, b}(z), Q(z)\right) t^{\frac{k_{4}+k_{5}}{2}+k_{6}} \\
\text { if } k_{4}<k_{5} \text { and } k_{4}+k_{5} \equiv 0(\bmod 2) \\
\lambda_{\kappa}^{(2)} D_{\frac{k_{4}-k_{5}}{2}, k_{5}, k_{6}}\left(a(z) a(z)^{T}, P_{a, b}(z), Q(z)\right) t^{\frac{k_{4}+k_{5}}{2}+k_{6}} \\
\text { if } k_{4}>k_{5} \text { and } k_{4}+k_{5} \equiv 0(\bmod 2) \\
\lambda_{\kappa}^{(3)} D_{k_{5}, k_{6}}\left(P_{a, b}(z), Q(z)\right) t^{k_{5}+k_{6}} \\
\text { if } k_{4}=k_{5} \text { and } k_{5} \equiv 0(\bmod 2) \\
0 \text { if } k_{4}+k_{5} \equiv 1(\bmod 2)
\end{array}\right.
$$

where $D_{n_{1}, \ldots, n_{i}}\left(A_{1}, \ldots, A_{i}\right)$ denotes the normalized Davis polynomials [5, 12 . and

$$
\begin{aligned}
P_{a, b}(z) & =\frac{a(z) b(z)^{T}+b(z) a(z)^{T}}{2}, \\
\lambda_{\kappa}^{(1)} & =2^{\frac{k_{4}+k_{5}}{2}+k_{6}-1} k_{4} k_{6}\left(k_{5}-k_{4}\right), \\
\lambda_{\kappa}^{(2)} & =2^{\frac{k_{4}+k_{5}}{2}+k_{6}-1} k_{5} k_{6}\left(k_{4}-k_{5}\right), \\
\lambda_{\kappa}^{(3)} & =2^{k_{5}+k_{6}} k_{5} k_{6} .
\end{aligned}
$$

Finally, the result holds.

The normalized Davis polynomials $D_{n_{1}, \ldots, n_{i}}\left(A_{1}, \ldots, A_{i}\right)$ can be computed efficiently using recursion formulas, see [12].

From Proposition 2, we obtain an upper bound of $\mathbb{E} \sup _{0 \leqslant t \leqslant \tau}\left\|\tilde{X}_{t, z}\right\|^{2 q}$ using the following lemma.

Lemma 2. For every martingale $Y_{t} \in L^{p}$ (where $p>1$ ), we have:

$$
\mathbb{E}\left[\sup _{0 \leqslant t \leqslant \tau}\left\|Y_{t}\right\|^{p}\right] \leqslant\left(\frac{p}{p-1}\right)^{p} \mathbb{E}\left[\left\|Y_{\tau}\right\|^{p}\right]
$$

Proof. See [1]. 
We thus have:

$$
\begin{aligned}
& \mathbb{E} \sup _{0 \leqslant t \leqslant \tau}\left\|\tilde{X}_{t, z}\right\|^{2 q} \leqslant \\
& \quad\left(\frac{2 q}{2 q-1}\right)^{2 q} \sum_{|\mathbf{k}|=q}\left(\begin{array}{l}
q \\
\mathbf{k}
\end{array}\right) C_{k_{1}, k_{2}, k_{3}}(z) \mu_{k_{4}, k_{5}, k_{6}}(z, 1) t^{k_{2}+2 k_{3}+k_{4}+\frac{k_{4}+k_{5}}{2}+k_{6}}
\end{aligned}
$$

where $C_{k_{1}, k_{2}, k_{3}}(z)$ and $\mu_{k_{4}, k_{5}, k_{6}}(z, 1)$ are given in Proposition 2 The computation of this deterministic upper-bound is much faster than the direct MonteCarlo approximation of the expectation. Furthermore, we get rid of the approximations inherent to the use of Monte-Carlo simulations.

\subsection{Probabilistic reachability}

Let us suppose for simplicity that $R$ is a ball of the form $B(O, \rho)$ where $O$ is the origin and $\rho>0$. Suppose that $x_{0} \in B\left(z, \delta_{0}\right)$. We have:

$$
\operatorname{Pr}\left(X_{\tau, x_{0}} \notin R\right)=\operatorname{Pr}\left(\left\|X_{\tau, x_{0}}\right\|>\rho\right) .
$$

Furthermore:

Proposition 3. $\operatorname{Pr}\left(X_{\tau, x_{0}} \in R\right) \geqslant 1-\frac{1}{\rho^{2}}\left(\delta_{\tau, \delta_{0}}+\sqrt{\mathbb{E}\left[\left\|\tilde{X}_{\tau, z}\right\|^{2}\right]}\right)^{2}$.

Proof. Using Chebyshev's inequality and triangular inequality, it follows

$$
\operatorname{Pr}\left(X_{\tau, x_{0}} \notin R\right) \leqslant \frac{1}{\rho^{2}} \mathbb{E}\left[\left\|X_{\tau, x_{0}}\right\|^{2}\right] \leqslant \frac{1}{\rho^{2}}\left(\sqrt{\mathbb{E}\left[\left\|\tilde{X}_{\tau, z}-X_{\tau, x_{0}}\right\|^{2}\right]}+\sqrt{\mathbb{E}\left[\left\|\tilde{X}_{\tau, z}\right\|^{2}\right]}\right)^{2} .
$$

We thus get, using the inequality of the previous section (namely, $\mathbb{E}\left[\| \tilde{X}_{\tau, z}-\right.$ $\left.X_{\tau, x_{0}} \|^{2} \leqslant \delta_{\tau, \delta_{0}}^{2}\right)$ :

$$
\operatorname{Pr}\left(X_{\tau, x_{0}} \notin R\right) \leqslant \frac{1}{\rho^{2}}\left(\delta_{\tau, \delta_{0}}\right)^{2}+\sqrt{\mathbb{E}\left[\left\|\tilde{X}_{\tau, z}\right\|^{2}\right]} .
$$

Alternatively, we have:

$$
\operatorname{Pr}\left(X_{\tau, x_{0}} \in R\right) \geqslant 1-\frac{1}{\rho^{2}}\left(\delta_{\tau, \delta_{0}}+\sqrt{\mathbb{E}\left[\left\|\tilde{X}_{\tau, z}\right\|^{2}\right]}\right)^{2} .
$$

NB: If we have $R=B\left(O^{\prime}, \rho\right)$ where $O^{\prime}$ is not the origin, we have:

$$
\operatorname{Pr}\left(X_{\tau, x_{0}} \in R\right) \geqslant 1-\frac{1}{\rho^{2}}\left(\delta_{\tau, \delta_{0}}+\sqrt{\mathbb{E}\left[\left\|\tilde{X}_{\tau, z}-O^{\prime}\right\|^{2}\right]}\right)^{2} .
$$




\subsection{Implementation}

This method has been implemented in the interpreted language Octave, and the experiments performed on a $2.80 \mathrm{GHz}$ Intel Core i7-4810MQ CPU with 8 GB of memory. The implementation is an adaptation of the program described in ([15) for controlling deterministic switched systems, but makes use of the tamed Euler scheme for SDEs (with the error function $\delta_{t, \delta_{0}}$ given in Theorem 2) instead of the classical Euler scheme.

Example 2. Consider the system of Example 1, for mode $u=1$ and $w=0.05$ :

$\mathrm{d} x_{1}=\left(-0.25 x_{1}+x_{2}+0.25\right) \mathrm{d} t+0.05 x_{1} \mathrm{~d} W_{t}^{1}$

$\mathrm{d} x_{2}=\left(-2 x_{1}-0.25 x_{2}-2\right) \mathrm{d} t+0.05 x_{2} \mathrm{~d} W_{t}^{2}$

The program gives (for $\tau=1, \Delta_{t}=\tau / 10^{4}$ ): $q=0, D=1.36, L_{g}=0.05$, $\lambda=0.25 ;$ and for $z=(-4,-3.8): E_{2, z}=893.3, E_{4, z}=2.14 \cdot 10^{5}, F_{2, z}=0.002$, $F_{4, z}=4.9 \cdot 10^{-6}$.

Consider now the system corresponding to Example 1 for mode $u=2$ and $w=0.05$ :

$\mathrm{d} x_{1}=\left(-0.25 x_{1}+2 x_{2}-0.25\right) \mathrm{d} t+0.05 x_{1} \mathrm{~d} W_{t}^{1}$

$\mathrm{d} x_{2}=\left(-x_{1}-0.25 x_{2}+1\right) \mathrm{d} t+0.05 x_{2} \mathrm{~d} W_{t}^{2}$

The program gives (for $\tau=1, \Delta_{t}=\tau / 10^{4}$ ): $q=0, D=1.36, L_{g}=0.05$, $\lambda=0.25$, and, for $z=(0,3): E_{2, z}=543.2, E_{4, z}=7.94 \cdot 10^{4}, F_{2, z}=0.0442$, $F_{4, z}=0.00178$.

Both computations take less than 10 s of $C P U$ time. Simulations of the two systems are given in Figure 1 for mode $u=1$ and starting point $z=(-4,3.8)$, and mode $u=2$ and starting point $z=(0,3)$. The initial ball $B\left(z, \delta_{0}\right)$ is depicted in black, the final ball $B\left(\mathbb{E}\left[\tilde{X}_{\tau, z}\right], \delta_{\tau, \delta_{0}}\right)$ in red, and 200 random sampling trajectories in blue for $t \in[0, \tau]$.

Example 3. We now consider a nonlinear model of a pendulum on a cart borrowed from [4, 31]. The dynamics is given by:

$\mathrm{d} x_{1}=x_{2} \mathrm{~d} t+0.03 x_{1} \mathrm{~d} W_{t}^{1}$

$\mathrm{d} x_{2}=\left(-\frac{g}{l} \sin x_{1}-\frac{k}{m} x_{2}+\frac{1}{m l^{2}} u\right) \mathrm{d} t+0.03 x_{2} \mathrm{~d} W_{t}^{2}$

where the state $\left(x_{1}, x_{2}\right)$ represents the angular position and velocity of the point mass on the pendulum, $u$ is the control input (torque applied to the cart). The parameters are $g=9.8, l=0.5, m=0.6, k=2$. The program gives for control input $u=0.5, \tau=0.1, \Delta_{t}=\tau / 10^{2}: q=1, D=2.56, L_{g}=0.03$, $\lambda=1.446 ;$ and for $z=(0,0): E_{2, z}=47.5, E_{4, z}=1977.2 \cdot 10^{5}, F_{2, z}=9.8 \cdot 10^{-4}$, $F_{4, z}=9.73 \cdot 10^{-7}$.

The computation takes less than 10 s of CPU time. Simulations of the system are given in Figure 2 for control input $u=0.5$ and starting point $z=(0,0)$. The initial ball $B\left(z, \delta_{0}\right)$ is depicted in black, the final ball $B\left(\mathbb{E}\left[\tilde{X}_{\tau, z}\right], \delta_{\tau, \delta_{0}}\right)$ in red, and 200 random sampling trajectories in blue for $t \in[0, \tau]$. 

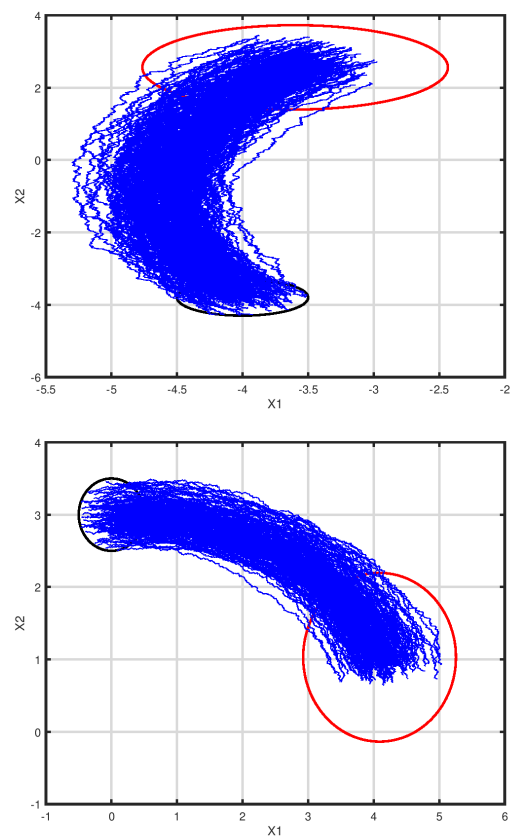

Figure 1: Simulations of Example 2 with mode $u=1$ and initial ball $B((-4,3.8), 0.5)$, and mode $u=2$ and initial ball $B((0,3), 0.5) ; \tau=1$.

\section{Sampled stochastic switched systems}

\subsection{Stochastic switched system as a finite collection of SDEs}

We now consider a finite number of SDEs. Each SDE is referred to as a mode $j$, and the set of modes is referred to as $U=\{1, \ldots, M\}$. We will denote by $X_{t, x_{0}}^{j}$ the solution at time $t$ of the system:

$$
\begin{aligned}
\mathrm{d} x(t) & =f_{j}(x(t))+g_{j}(x(t)) \mathrm{d} W_{t}^{j}, \\
x(0) & =x_{0} .
\end{aligned}
$$

where $x_{0}$ is a random variable that is measurable in $\mathcal{F}_{0}$. Hypotheses (H1)(H2)-( $(\mathrm{H} 3)$, as defined in Section 3 , are naturally extended to every mode $j$ of $U$. Accordingly, constants $L_{g}, \lambda, F$ associated to SDE (1) in Section 3 , now become $L_{g_{j}}, \lambda_{j}, F_{j}$ respectively, for each $j \in U$.

Likewise, for each $j \in U$, the nonnegative real $\left(\delta_{t, \delta_{0}}\right)^{2}$ becomes $\left(\delta_{t, \delta_{0}}^{j}\right)^{2}$ for each mode $j$; the approximate continuous-time solution of $[19)$ starting from $z$, is denoted by $\tilde{X}_{t, z}^{j}$, and the approximate staircase solution by $\underline{X}_{t, z}^{j}$.

\subsection{Control patterns}

The control laws that we now consider are "piecewise constant of duration $\tau$ " in the sense that, every $\tau$ seconds, they select a given mode (see ([30])). We call 


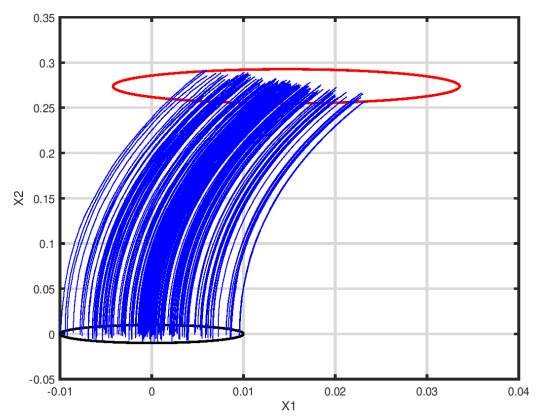

Figure 2: Simulations of Example 3 with control input $u=0.5$, initial ball $B((0,0), 0.01)$, $\tau=0.1$.

"(control) pattern of length $k$ " a sequence of $k$ modes (i.e., an element of $U^{k}$ ). Each pattern $\pi$ of the form $j_{1} j_{2} \cdots j_{k}$ corresponds to the selection of mode $j_{1}$ for time $t \in[0, \tau)$, then mode $j_{2}$ for $t \in[\tau, 2 \tau)$, and so on, until $t=k \tau$. We assume that the solution of the system is continuous at sampling instants $t=\tau, 2 \tau, \ldots$ (which means that there is no "reset" of the system at sampling instants).

Given a stochastic switched system, a pattern $\pi$ of length $k$ and an initial random variable $z$, one constructs the "approximate solution controlled by $\pi$ " by composing together the approximations obtained by successive application of the modes of the pattern $\pi$. Formally, the "continuous" approximate solution $\tilde{X}_{t, z}^{\pi}$ is defined at time $t \in[0, k \tau]$ as follows:

- $\tilde{X}_{t, z}^{\pi}=\tilde{X}_{t, z}^{j}$ if $\pi=j \in U, k=1$ and $t \in[0, \tau]$, and

- $\tilde{X}_{(k-1) \tau+t^{\prime}, z}^{\pi}=\tilde{X}_{t, z^{\prime}}^{j}$ with $z^{\prime}=\tilde{X}_{(k-1) \tau, z}^{\pi^{\prime}}$ if $k \geqslant 2, t^{\prime} \in[0, \tau], \pi=\pi^{\prime} * j$ for some $j \in U$ and $\pi^{\prime} \in U^{k-1}$.

The "staircase" approximate solution $\underline{X}_{t, z}^{\pi}$ is defined analogously. Likewise, given an initial error radius $\delta_{0}>0$ and a pattern $\pi$ of length $k \geqslant 1$, one defines the error radius $\delta_{t, \delta_{0}}^{\pi}$ using (7) as follows:

- $\delta_{t, \delta_{0}}^{\pi}=\delta_{t, \delta_{0}}^{j}$ if $\pi=j \in U, k=1$ and $t \in[0, \tau]$, and

- $\delta_{(k-1) \tau+t^{\prime}, \delta_{0}}^{\pi}=\delta_{t^{\prime}, \delta^{\prime}}^{j}$ with $\delta^{\prime}=\delta_{(k-1) \tau, \delta_{0}}^{\pi^{\prime}}$, if $k \geqslant 2, t^{\prime} \in[0, \tau], \pi=\pi^{\prime} * j$ for some $j \in U$ and $\pi^{\prime} \in U^{k-1}$.

\subsection{Controlled probabilistic reachability}

Given a ball $R=B(O, \rho) \subset \mathbb{R}^{d}$ with $\rho>0$, we define the problem of "controlled probabilistic reachability inside $R$ " as follows:

Given a starting point $z \in \mathbb{R}^{d}$ and a positive real $\delta_{0}$, find a pattern $\pi$ of length $k$ such that, at $t=k \tau$, the image of the points located initially in $B\left(z, \delta_{0}\right)$ be, on average, located as close as possible to $O$, and, in particular:

$$
B\left(\mathbb{E}\left[\tilde{X}_{t, z}^{\pi}\right], \delta_{t, \delta_{0}}^{\pi}\right) \subseteq R \quad \text { for } t=k \tau .
$$


The program mentioned in Section 3.5. has been extended in order to find, by exhaustive enumeration ${ }^{1}$, such a pattern $\pi$. This guarantees that, for all $x_{0} \in B\left(z, \delta_{0}\right)$ :

$$
\operatorname{Pr}\left(X_{k \tau, x_{0}}^{\pi} \in R\right) \geqslant 1-\frac{1}{\rho^{2}}\left(\sqrt{\mathbb{E}\left[\left\|\tilde{X}_{k \tau, z}^{\pi}\right\|^{2}\right]}+\delta_{k \tau, \delta_{0}}^{\pi}\right)^{2},
$$

We now give two examples of application of this program.

Example 4. Consider the system of Example 1 with $w=0.01$ :

$$
\begin{aligned}
& \mathrm{d} x_{1}=\left(-0.25 x_{1}+u x_{2}+(-1)^{u} 0.25\right) \mathrm{d} t+0.01 x_{1} \mathrm{~d} W_{t}^{1} \\
& \mathrm{~d} x_{2}=\left((u-3) x_{1}-0.25 x_{2}+(-1)^{u}(3-u)\right) \mathrm{d} t+0.01 x_{2} \mathrm{~d} W_{t}^{2}
\end{aligned}
$$

where $u=1,2$.

For $\tau=0.5, \Delta_{t}=10^{-4}$, one finds (for all modes $u=1,2$ ):

$q=0, D=1.36, L_{g}=0.01, \lambda=0.25 ;$ for $z=(-4,-3.8): E_{2, z}=893.31$, $E_{4, z}=2.14 \cdot 10^{5}, F_{2, z}=0.002, F_{4, z}=4.9 \cdot 10^{-6} ;$ and for $z=(0,3): E_{2, z}=$ $543.22, E_{4, z}=7.94 \cdot 10^{4}, F_{2, z}=0.0442, F_{4, z}=0.00178$.

Our program shows the probabilistic reachability inside $R=B((0,0), \rho)$, for all point $x_{0} \in B\left((5,4), \delta_{0}\right)$ and all point $y_{0} \in B\left((-5,-4), \delta_{0}\right)$, with $\delta_{0}=0.1$ and $\rho=7$. We have, for $\pi_{1}=(2 \cdot 2 \cdot 2 \cdot 2)$

$$
\begin{gathered}
\operatorname{Pr}\left(X_{4 \tau, x_{0}}^{\pi_{1}} \in R\right) \geqslant 1-\frac{1}{\rho^{2}}\left(\sqrt{\mathbb{E}\left[\left\|\tilde{X}_{4 \tau,(5,4)}^{\pi_{1}}\right\|^{2}\right]}+\delta_{4 \tau, \delta_{0}}^{\pi_{1}}\right)^{2} \\
\operatorname{Pr}\left(X_{4 \tau, x_{0}}^{\pi_{1}} \in R\right) \geqslant 0.689
\end{gathered}
$$

and for $\pi_{2}=(1 \cdot 1 \cdot 1 \cdot 1 \cdot 1)$ :

$$
\begin{gathered}
\operatorname{Pr}\left(X_{5 \tau, y_{0}}^{\pi_{2}} \in R\right) \geqslant 1-\frac{1}{\rho^{2}}\left(\sqrt{\mathbb{E}\left[\left\|\tilde{X}_{5 \tau,(-5,-4)}^{\pi_{2}}\right\|^{2}\right]}+\delta_{5 \tau, \delta_{0}}^{\pi_{2}}\right)^{2} . \\
\operatorname{Pr}\left(X_{5 \tau, y_{0}}^{\pi_{2}} \in R\right) \geqslant 0.713
\end{gathered}
$$

Figure 3 depicts in black the initial balls (at $t=0)$ centered at $(5,4)$ and $(-5,-4)$; and for each initial ball, the pattern that sends the ball inside $R$ (at time $t=k \tau)$; the intermediate balls (at $t=\tau, 2 \tau, \ldots,(k-1) \tau)$ are depicted in red, and 200 sampling trajectories drawn in black.

Example 5. (the slit problem)

The problem is adapted from ([20]). The controlled dynamics is:

$$
\mathrm{d} X=u \mathrm{~d} t+\mathrm{d} W, \quad X_{0}=1
$$

with mode $u \in\{-6,-5,-4,-3,-2,1,0,1,2,3,4,5,6\}$. We have (at $t=0.5$ ) a slit at $x \in[-1,-3]$. The objective is thus to control the system so that $x(t) \in$ $R=[-1,-3]$ (i.e., $x(t) \in R=B\left(O^{\prime}, \rho\right)$ with $O^{\prime}=-2$ and $\rho=1$ ) at $t=0.5$.

\footnotetext{
${ }^{1}$ In Section 4.4 a strategy based on the search of an optimal pattern is suggested rather than an exhaustive search.
} 

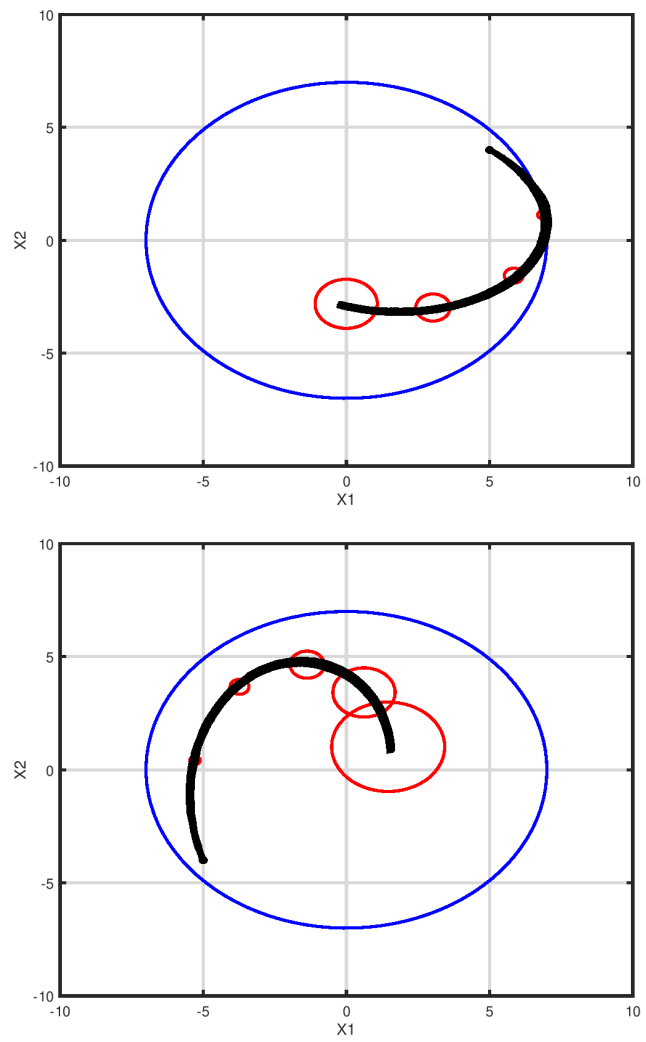

Figure 3: Simulations of Example 4 from the initial balls $B((5,4), 0.1)$ and $B((-5,-4), 0.1)$ using patterns $(2 \cdot 2 \cdot 2 \cdot 2)$ and $(1 \cdot 1 \cdot 1 \cdot 1 \cdot 1)$ resp., with $\tau=0.5$.

One has, for all modes: $q=0, D=0, L_{g}=0, \lambda=0$. For $\delta_{0}=0.2$, an initial point $z=1$ and a sampling time $\tau=0.5$ with subsampling $\Delta_{t}=2.5 \times 10^{-3}$, one has for mode $u=-6: E_{2, z}=144, E_{4, z}=20736, F_{2, z}=4, F_{4, z}=16$; and for mode $u=0: E_{2, z}=0, E_{4, z}=0, F_{2, z}=4, F_{4, z}=16$.

Suppose that all the trajectories start at $x_{0} \in B\left(z, \delta_{0}\right)$, with $z=1$ and $\delta_{0}=0.2$. When there is no control $(u=0)$, at time $t=0.5$, the expected value of $X_{t, x_{0}}$ is in $B\left(z_{1}, \delta_{t, \delta_{0}}\right)$ with $z_{1}=1$ and $\delta_{t, \delta_{0}}=0.29$. From Markov's inequality, it follows that the trajectories pass by $R=B(-2,1)$ at $t=0.5$ with low probability: see Figure 4. On the other hand, with control $u=-6$, at time $t=\tau=0.5$, the expected value of $X_{t, x_{0}}$ is now in $B\left(z_{1}^{\prime}, \delta_{\tau, \delta_{0}}^{\prime}\right)$ with $z_{1}^{\prime}=-2$ and $\delta_{\tau, \delta_{0}}^{\prime}=0.29$. This explains why the trajectories pass by $R=B(-2,1)$ at $t=0.5$ with high probability. We have, for $x_{0} \in B\left(z, \delta_{0}\right)$ with $z=1, \delta_{0}=0.2$, $t=\tau=0.5, \rho=1, O^{\prime}=-2$ :

$$
\operatorname{Pr}\left(X_{\tau, x_{0}}^{u} \in R=B\left(O^{\prime}, \rho\right)\right) \geqslant 1-\frac{1}{\rho^{2}}\left(\sqrt{\mathbb{E}\left[\left\|\tilde{X}_{\tau, z}^{u}-O^{\prime}\right\|^{2}\right]}+\delta_{\tau, \delta_{0}}^{u}\right)^{2} .
$$



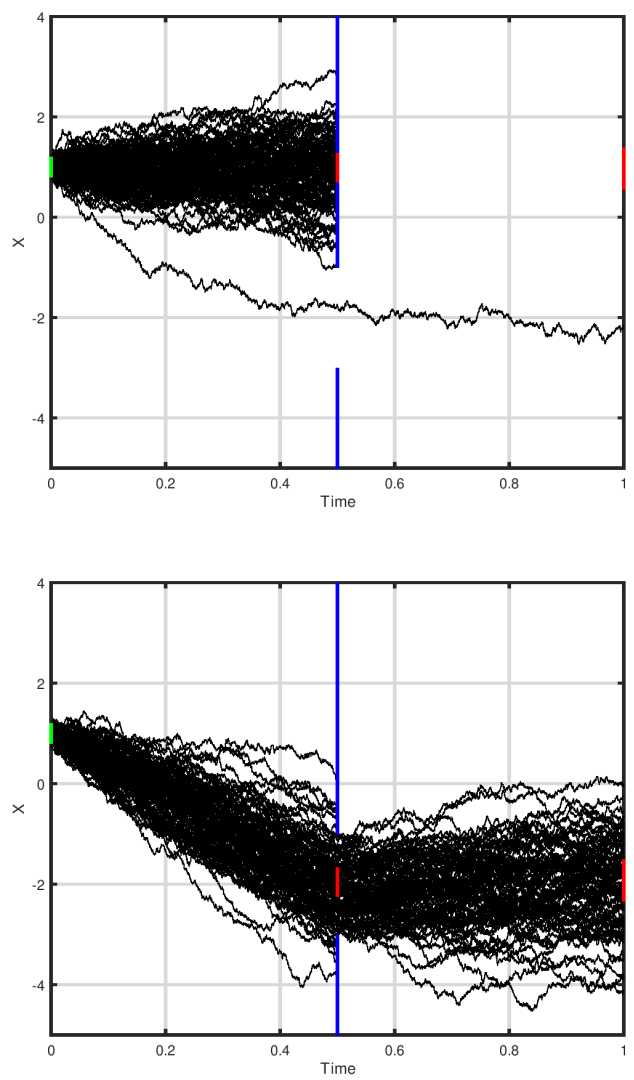

Figure 4: Simulations of Example 5 without control $(\pi=(0 \cdot 0))$ and with control pattern $(-6 \cdot 0)$ from the initial ball $B(1,0.2)$. The initial region is in green. The red regions are $B\left(\mathbb{E}\left[\tilde{X}_{t, z}^{\pi}\right], \delta_{t, \delta_{0}}^{\pi}\right)$. with $z=1, \delta_{0}=0.2$ for the two patterns $\pi=(0 \cdot 0)$ (top) and $\pi=(-6 \cdot 0)$ (bottom).

For mode $u=-6$, this gives: $\delta_{\tau, \delta_{0}}^{u}=0.29$ and $\mathbb{E}\left[\left\|\tilde{X}_{\tau, z}^{u}-O^{\prime}\right\|^{2}\right]=0.048$. It follows: $\operatorname{Pr}\left(X_{\tau, x_{0}}^{u} \in R\right) \geqslant 0.871$.

\subsection{Optimal control by state space discretization}

In this Subsection, we are interested in solving an optimal control problem in order to minimize the average distance of the state reached after a given number of steps to the origin. In order to solve such optimal control problems, it is classical to spatially discretize the set $S$ into a finite number of cells of equal size. Consider a partition of a compact subset $S$ of $R^{d}$ in cells. For the sake of simplicity, we suppose that each cell $C$ is a hypercube of $R^{d}$ of side length $2 \varepsilon / \sqrt{d}$, and we consider the centre of the cell, denoted $\sharp x$, as the "representative" of the cell. Note that any point $z$ located in the cell is such that $\|z-\sharp x\| \leqslant \varepsilon$. In [3] (cf. [8]), it is explained how a discretized procedure, 
based on classical techniques of dynamic programming, constructs a mapping $\mathbf{u}$ which associates to each representative $\sharp x$ a mode $\mathbf{u}(\sharp x) \in U$, corresponding to the optimal policy at position $\sharp x$. More precisely, for any representative point $\sharp x_{0, \varepsilon}$, the discretized procedure $\operatorname{PROC}\left(\sharp x_{0, \varepsilon}\right)$ constructs a sequence of modes $u_{1, \varepsilon}, \ldots, u_{k, \varepsilon}$ and a sequence of representatives $\sharp x_{1, \varepsilon}, \ldots, \sharp x_{k, \varepsilon}$ such that, for all $1 \leqslant i \leqslant k$ :

- $u_{i, \varepsilon}=\mathbf{u}\left(\sharp x_{i-1, \varepsilon}\right)$.

- $\sharp x_{i, \varepsilon}$ is the representative of the cell containing $\mathbb{E}\left[\tilde{X}_{\tau, \sharp x_{i-1, \varepsilon}}^{u_{i, \varepsilon}}\right]$.

For the sake of notation simplicity, we will now drop the index $\varepsilon$ in symbols $u_{i, \varepsilon}$ and $\sharp x_{i, \varepsilon}$. Let us define the function $\operatorname{next}^{u_{i}}$ by: $\operatorname{next}^{u_{i}}\left(\sharp x_{i-1}\right)=\sharp x_{i}$, where $\sharp x_{i}$ is the representative of the cell containing $\mathbb{E}\left[\tilde{X}_{\tau, \sharp x_{i-1}}^{u_{i}}\right]$. We define the function $n e x t^{\pi}$ as follows:

- if $\pi=u$ for some $u \in U$ then $n \operatorname{ext} t^{\pi}(\sharp x)=n \operatorname{ext} t^{u}(\sharp x)$,

- if $\pi$ is of the form $u \cdot \pi^{\prime}$ then $\operatorname{next}^{\pi}(\sharp x)=\operatorname{next}^{\pi^{\prime}}\left(n \operatorname{ext}^{u}(\sharp x)\right)$.

Given a point $z_{0} \in \mathbb{R}^{d}$ and a positive integer $k$, we focus on the problem of finding the optimal pattern $\pi^{*}\left(z_{0}\right) \in U^{k}$, which, at time $T=k \tau$, maps $z_{0}$ to a distribution located at a minimal distance of the origin, i.e., such that:

$$
\pi^{*}\left(z_{0}\right)=\operatorname{argmin}_{\pi \in U^{k}} \mathbb{E}\left[\left\|X_{T, z_{0}}^{\pi}\right\|^{2}\right] \text {. }
$$

Let $\sharp x_{0}$ be the representative of $z_{0}$. The sequences $\left\{u_{i}\right\}_{1 \leqslant i \leqslant k}$ and $\left\{\sharp x_{i}\right\}_{1 \leqslant i \leqslant k}$ given by $\operatorname{PROC}\left(\sharp x_{0}\right)$, are optimal in the following sense: (see, e.g., [3, 8]):

- the pattern $u_{1} \cdots u_{k}$ coincides with $\pi^{*}\left(z_{0}\right)$ when $\varepsilon$ is sufficiently small, and

- the discrete random variable $\sharp x_{k}$ converges in distribution to the random variable $X_{T, z_{0}}^{u_{1} \cdots u_{k}}$ when $\varepsilon$ tends to 0 .

This justifies the employment of the pattern $u_{1} \cdots u_{k}$ generated by $\operatorname{PROC}\left(\sharp x_{0}\right)$ as a (sub)optimal control pattern $\pi$ for the original stochastic diffusion system 2

Let us now, in our context, give a precise upper bound to $\mathbb{E}\left[\left\|X_{T, z_{0}}^{u_{1} \cdots u_{k}}-\sharp x_{k}\right\|^{2}\right]$, where $\sharp x_{k}$ is the representative point defined by $n e x t^{u_{1} \cdots u_{k}}\left(\sharp x_{0}\right)$. We first define $\lambda_{i} \geqslant 0$, for $1 \leqslant i \leqslant k$ :

$$
\lambda_{i}:=\sqrt{\mathbb{E}\left[\left\|\tilde{X}_{\tau, \sharp x_{i-1}}^{u_{i}}-\sharp x_{i}\right\|^{2}\right]} .
$$

Let us also define $\Delta\left(u_{1} \cdots u_{k}\right)$ recursively by:

$$
\text { - } \Delta\left(u_{1} \cdots u_{i}\right)=\delta_{\tau, \lambda_{i-1}+\Delta\left(u_{1} \cdots u_{i-1}\right)}^{u_{i}} \text { for } i=2, \ldots, k \text {, and }
$$

\footnotetext{
${ }^{2}$ see, e.g., 3
} 
- $\Delta\left(u_{1}\right)=\delta_{\tau, \varepsilon}^{u_{1}}$.

Without loss of understanding, we abbreviate $\Delta\left(u_{1} \cdots u_{i}\right)$ by $\Delta_{i}$. We have

Lemma 3. $\sqrt{\mathbb{E}\left[\left\|X_{k \tau, z_{0}}^{u_{1} \cdots u_{k}}-\tilde{X}_{\tau, \sharp x_{k-1}}^{u_{k}}\right\|^{2}\right]} \leqslant \Delta_{k}$.

Proof. The proof is by induction on $k$. The case $k=1$ is trivial. Suppose $k \geqslant 2$. We have:

$$
\sqrt{\mathbb{E}\left[\left\|X_{k \tau, z_{0}}^{u_{1} \cdots u_{k}}-\tilde{X}_{\tau, \sharp x_{k-1}}^{u_{k}}\right\|^{2}\right]} \leqslant \delta_{\tau, \sqrt{\mathbb{E}\left[\left\|X_{(k-1) \tau, z_{0}}^{u_{1} \cdots u_{k-1}}-\sharp x_{k-1}\right\|^{2}\right]}}^{u_{k}}
$$

On the other hand, using the triangular inequality and the induction hypothesis, we have:

$$
\begin{aligned}
& \sqrt{\mathbb{E}\left[\left\|X_{(k-1) \tau, z_{0}}^{u_{1} \cdots u_{k-1}}-\sharp x_{k-1}\right\|^{2}\right]} \\
& \leqslant \sqrt{\mathbb{E}\left[\left\|X_{(k-1) \tau, z_{0}}^{u_{1} \cdots u_{k-1}}-\tilde{X}_{\tau, \sharp x_{k-2}}^{u_{k-1}}\right\|^{2}\right]}+\sqrt{\mathbb{E}\left[\left\|\tilde{X}_{\tau, \sharp x_{k-2}}^{u_{k-1}}-\sharp x_{k-1}\right\|^{2}\right]} \\
& \leqslant \Delta_{k-1}+\lambda_{k-1} .
\end{aligned}
$$

Hence: $\sqrt{\mathbb{E}\left[\left\|X_{k \tau, z_{0}}^{u_{1} \cdots u_{k}}-\tilde{X}_{\tau, \sharp x_{k-1}}^{u_{k}}\right\|^{2}\right]} \leqslant \delta_{\tau, \Delta_{k-1}+\lambda_{k-1}}^{u_{k}}=\Delta_{k}$. This achieves the proof of the induction step.

We have

Theorem 3. Consider a point $z_{0} \in \mathbb{R}^{d}$ of representative $\sharp x_{0}$, and let $\sharp x_{k}=$ next ${ }^{u_{1} \cdots u_{k}}\left(\sharp x_{0}\right)$ for sequence $u_{1} \cdots u_{k} \in U^{k}$ (generated by PROC $\left(\sharp x_{0}\right)$ ). We have:

$$
\sqrt{\mathbb{E}\left[\left\|X_{T, z_{0}}^{u_{1} \cdots u_{k}}-\sharp x_{k}\right\|^{2}\right]} \leqslant \Delta_{k}+\lambda_{k} .
$$

Proof. We have

1. $\mathbb{E}\left[\left\|X_{k \tau, z_{0}}^{u_{1} \cdots u_{k}}-\tilde{X}_{\tau, \sharp x_{k-1}}^{u_{k}}\right\|^{2}\right] \leqslant \Delta_{k}^{2}$ by Lemma 3 , and

2. $\mathbb{E}\left[\left\|\tilde{X}_{\tau, \sharp x_{k-1}}^{u_{k}}-\sharp x_{k}\right\|^{2}\right] \leqslant \lambda_{k}^{2}$ by definition.

It follows, using triangular inequality: $\sqrt{\mathbb{E}\left[\left\|X_{T, z_{0}}^{u_{1} \cdots u_{k}}-\sharp x_{k}\right\|^{2}\right]} \leqslant \Delta_{k}+\lambda_{k}$.

Note that the term $\Delta_{k}$ of the inequality right-hand side takes into account the error introduced by the Euler scheme (with respect to the real solution of the SDE); in contrast, in the literature (e.g. [8, 22]), the Euler error is often disregarded, the analysis focusing just on the convergence of $\sharp x_{k}$ to the Euler approximation $\tilde{X}_{T, z_{0}}^{u_{1} \cdots u_{k}}$.

Example 6. We apply the above procedure PROC $\left.\sharp x_{0}\right)$ on Example 1 with $w=0.01$. Consider the interest set $R=B((0,0), \rho)$ with $\rho=7$, discretized with an accuracy $\varepsilon=0.57$. We compute (sub)optimal patterns for the entire set, using different lengths of patterns, and simulate the induced controller for 200 initial conditions randomly selected in $R$. Simulations are given in Figure 5. The procedure took 11.8 seconds of computation for patterns of length $1,47.4$ seconds of computation for patterns of length $3,152.0$ seconds of computation for patterns of length 5 . 

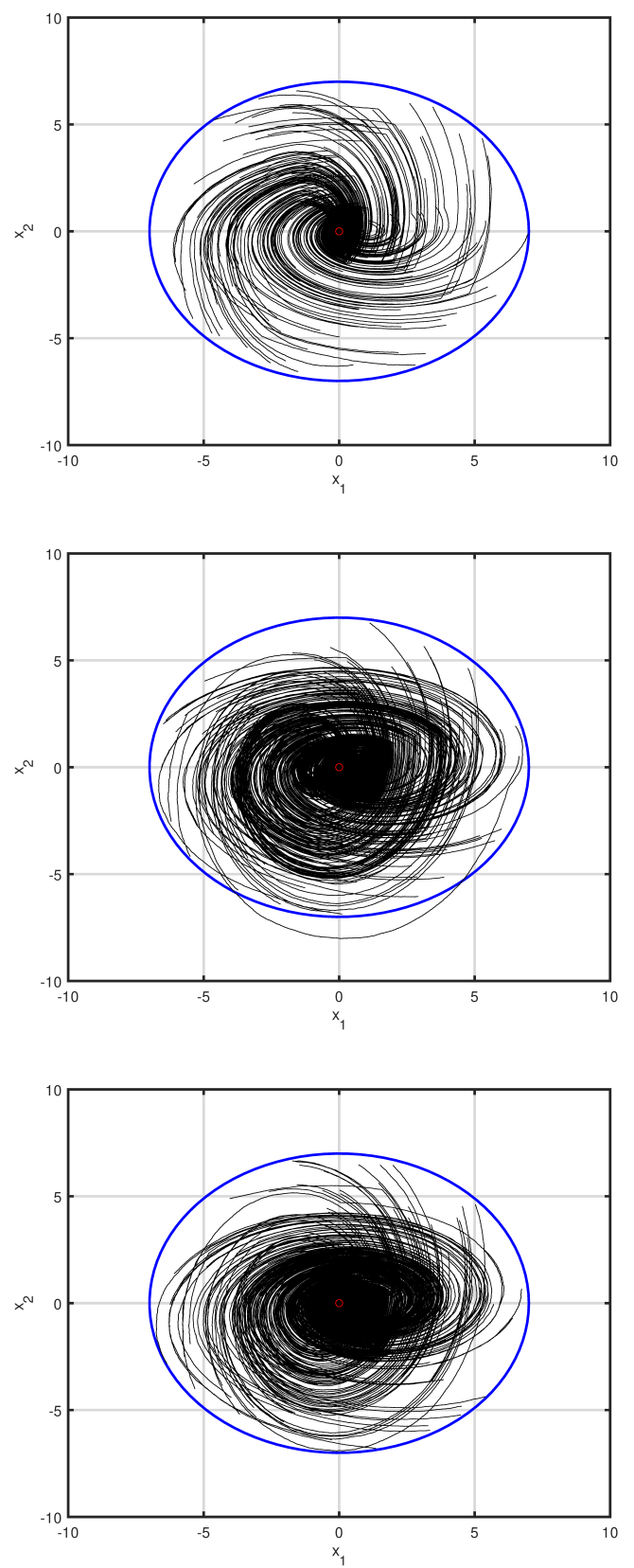

Figure 5: Simulations of Example 6 with the controller induced by $\operatorname{PROC}\left(\sharp x_{0}\right)$, for patterns of length 1 (top), length 3 (center), and length 5 (bottom). The blue circle is the set $R=$ $B((0,0), 7)$, the red marker is the target state (the origin), the black lines are the controlled trajectories. 
We now compare the results yielded by PROC $\left(\sharp x_{0}\right)$ with those of Example 4. The sub-optimal procedure returns patterns ensuring the probabilistic reachability inside $R=B((0,0), \rho)$, for all point $x_{0} \in B\left((5,4), \delta_{0}\right)$ and all point $y_{0} \in B\left((-5,-4), \delta_{0}\right)$, with $\delta_{0}=0.1$ and $\rho=7$. For $x_{0} \in B\left((5,4), \delta_{0}\right)$, the procedure returns pattern $\pi_{1}=(1 \cdot 2 \cdot 2 \cdot 2)$ ensuring a probability

$$
\operatorname{Pr}\left(X_{4 \tau, x_{0}}^{\pi_{1}} \in R\right) \geqslant 0.801
$$

and for $x_{0} \in B\left((-5,-4), \delta_{0}\right)$, the procedure returns pattern $\pi_{2}=(2 \cdot 2 \cdot 2 \cdot 1 \cdot 1)$, with probability

$$
\operatorname{Pr}\left(X_{5 \tau, y_{0}}^{\pi_{2}} \in R\right) \geqslant 0.802 .
$$

The probabilities are thus higher than in Example 4. Simulations are shown in Figure 6, depicting in black the initial balls $($ at $t=0)$ centered at $(5,4)$ and $(-5,-4)$; and for each initial ball, the pattern that sends the ball inside $R$ (at time $t=k \tau)$; the intermediate balls (at $t=\tau, 2 \tau, \ldots,(k-1) \tau$ ) are depicted in red, and 200 sampling trajectories drawn in black.
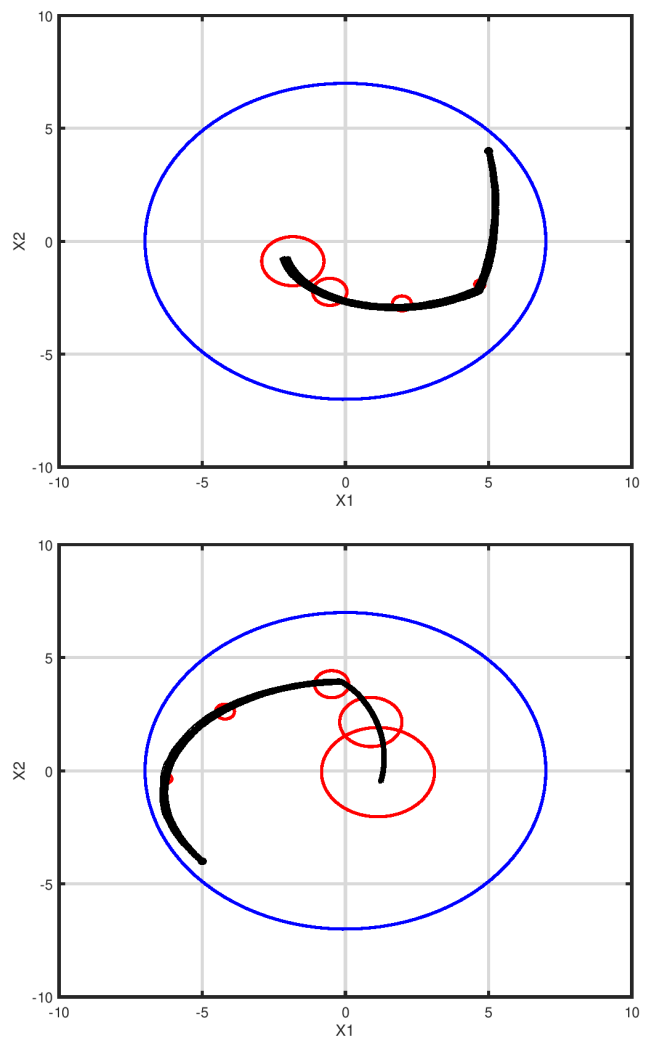

Figure 6: Simulations of Example 6 from the initial balls $B((5,4), 0.1)$ and $B((-5,-4), 0.1)$ using patterns $(1 \cdot 2 \cdot 2 \cdot 2)$ and $(2 \cdot 2 \cdot 2 \cdot 1 \cdot 1)$ resp., with $\tau=0.5$. 
Example 7. We consider nonlinear Example 3. Let us recall the dynamics: $\mathrm{d} x_{1}=x_{2} \mathrm{~d} t+0.03 x_{1} \mathrm{~d} W_{t}^{1}$

$\mathrm{d} x_{2}=\left(-\frac{g}{l} \sin x_{1}-\frac{k}{m} x_{2}+\frac{1}{m l^{2}} u\right) \mathrm{d} t+0.03 x_{2} \mathrm{~d} W_{t}^{2}$

We consider the set of control inputs $u \in\{-1.5,-1,-0.5,0,0.5,1,1.5\}$. In order to reproduce the results of [31], we slightly modify the procedure so that the interest set is $R=B((0,0), \rho)$ with $\rho=1.0$, but the optimization objective is to minimize the average distance with the target state $x^{o b j}=(0.5,0)$. The state space is discretized with an accuracy $\varepsilon=0.02$. We compute (sub)optimal patterns for the entire set $R$, using patterns of length 1 , and simulate the induced controller for 30 initial conditions randomly selected in $R$. Simulations are given in Figure 7 . The procedure took 127 seconds of computation for patterns of length 1.

\section{Final remarks and future work}

We have established a mean-square error bound on an Euler-like method to simulate stochastic differential equations that satisfy a one-sided Lipschitz (OSL) condition. An advantage of our method is that the OSL assumption is weaker than the condition under which classical methods converge. From the mean square error bound, we have inferred a lower bound on the probability to reach a given set $R$ at a given time. This allows us to determine a control pattern (in term of sequences of control modes) which makes the stochastic switched system reach a target with guaranteed minimum probability. Besides, instead of searching over all possible sequences of modes (which is a process exponential in the length of sequences), we have proposed an optimized method inferring a 'best' pattern, which may entail a significant gain in time and space. In the future, we plan to experiment our method with significantly larger examples than those given here.

\section{References}

[1] Ludwig Arnold. Stochastic differential equations. New York, 1974.

[2] Eugene Asarin, Olivier Bournez, Thao Dang, Oded Maler, and Amir Pnueli. Effective synthesis of switching controllers for linear systems. Proceedings of the IEEE, 88(7):1011-1025, 2000.

[3] D Bertsekas. Convergence of discretization procedures in dynamic programming. IEEE Transactions on Automatic Control, 20(3):415-419, 1975.

[4] Alessandro Borri, Giordano Pola, and Maria Domenica Di Benedetto. Symbolic models for nonlinear control systems affected by disturbances. International Journal of Control, 85(10):1422-1432, 2012.

[5] AW Davis. On the construction of a class of invariant polynomials in several matrices, extending the zonal polynomials. Annals of the Institute of Statistical Mathematics, 33(1):297-313, 1981. 
[6] Tzanko Donchev and Elza Farkhi. Stability and euler approximation of one-sided lipschitz differential inclusions. SIAM journal on control and optimization, 36(2):780-796, 1998.

[7] S Esmaeil Zadeh Soudjani. Formal abstractions for automated verification and synthesis of stochastic systems. 2014.

[8] Sadegh Esmaeil Zadeh Soudjani and Alessandro Abate. Adaptive and sequential gridding procedures for the abstraction and verification of stochastic processes. SIAM Journal on Applied Dynamical Systems, 12(2):921-956, 2013.

[9] L. Fribourg, U. Kühne, and R. Soulat. Finite controlled invariants for sampled switched systems. Formal Methods in System Design, 45(3):303329 , Dec 2014.

[10] Antoine Girard, Giordano Pola, and Paulo Tabuada. Approximately bisimilar symbolic models for incrementally stable switched systems. IEEE Transactions on Automatic Control, 55(1):116-126, 2010.

[11] Desmond J. Higham, Xuerong Mao, and Andrew M. Stuart. Strong convergence of Euler-type methods for nonlinear stochastic differential equations. SIAM Journal on Numerical Analysis, 40(3):1041-1063, 2002.

[12] Grant Hillier, Raymond Kan, and Xiaolu Wang. Generating functions and short recursions, with applications to the moments of quadratic forms in noncentral normal vectors. Econometric Theory, 30(2):436-473, 2014.

[13] Martin Hutzenthaler, Arnulf Jentzen, and Peter E. Kloeden. Strong convergence of an explicit numerical method for SDEs with nonglobally lipschitz continuous coefficients. Annals of Applied Probability, 22(4):1611-1641, 2012 .

[14] Adrien Le Coënt, Julien Alexandre dit Sandretto, Alexandre Chapoutot, and Laurent Fribourg. An improved algorithm for the control synthesis of nonlinear sampled switched systems. Formal Methods in System Design, Nov 2016.

[15] Adrien Le Coënt, Florian De Vuyst, Ludovic Chamoin, and Laurent Fribourg. Control synthesis of nonlinear sampled switched systems using Euler's method. In Proceedings of 3rd Intl Workshop on Symbolic and Numerical Methods for Reachability Analysis (SNR 2017), 2017.

[16] Daniel Liberzon. Switching in systems and control. Springer Science \& Business Media, 2003.

[17] Jan R Magnus. The moments of products of quadratic forms in normal variables. Statistica Neerlandica, 32(4):201-210, 1978. 
[18] Kostas Margellos and John Lygeros. Hamilton-Jacobi formulation for reach-avoid differential games. IEEE Transactions on Automatic Control, 56(8):1849-1861, 2011.

[19] G. Maruyama. Continuous Markov processes and stochastic equations. Rend. Circ. Mat. Palermo, 2(4):48-90, 1955.

[20] Matthias Morzfeld. Implicit sampling for path integral control, Monte Carlo localization, and SLAM. Journal of Dynamic Systems, Measurement, and Control, 137(5), 2015.

[21] B.K. Oksendal. Stochastic Differential Equations: An Introduction with Applications. Springer, 2002.

[22] Huyên Pham et al. On some recent aspects of stochastic control and their applications. Probability Surveys, 2:506-549, 2005.

[23] Stephen Prajna. Barrier certificates for nonlinear model validation. Automatica, 42(1):117-126, 2006.

[24] Muhammad Zakiyullah Romdlony and Bayu Jayawardhana. Stabilization with guaranteed safety using control lyapunov-barrier function. Automatica, 66(C):39-47, 2016.

[25] S Soudjani, Caspar Gevaerts, and Alessandro Abate. Faust 2: Formal abstractions of uncountable-state stochastic processes. arXiv preprint arXiv:1403.3286, 2014.

[26] Sean Summers and John Lygeros. Verification of discrete time stochastic hybrid systems: A stochastic reach-avoid decision problem. Automatica, 46(12):1951 - 1961, 2010.

[27] Max-K. von Renesse and Michael Scheutzow. Existence and uniqueness of solutions of stochastic functional differential equations. Random Operators and Stochastic Equations, 18(3), 2010.

[28] Peter Wieland and Frank Allgöwer. Constructive safety using control barrier functions. In Proceedings of the 7th IFAC Symposium on Nonlinear Control Systems, pages 462-467, 2007.

[29] R. Wisniewski and M. L. Bujorianu. Stochastic safety analysis of stochastic hybrid systems. In 2017 IEEE 56th Annual Conference on Decision and Control, pages 2390-2395, 2017.

[30] Majid Zamani, Alessandro Abate, and Antoine Girard. Symbolic models for stochastic switched systems: A discretization and a discretization-free approach. Automatica, 55:183-196, 2015. 
[31] Majid Zamani, Peyman Mohajerin Esfahani, Rupak Majumdar, Alessandro Abate, and John Lygeros. Symbolic control of stochastic systems via approximately bisimilar finite abstractions. IEEE Trans. Automat. Contr., 59(12):3135-3150, 2014.

[32] Majid Zamani, Ilya Tkachev, and Alessandro Abate. Towards scalable synthesis of stochastic control systems. Discrete Event Dynamic Systems, 27(2):341-369, 2017. 


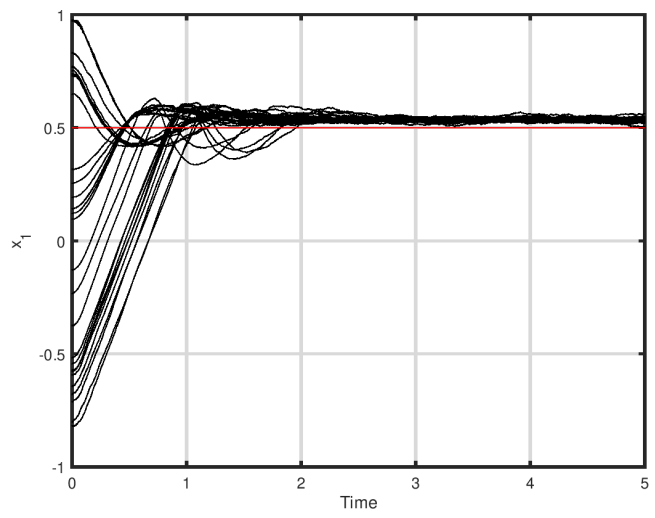

Figure 7: Simulations of Example 7 projected on the first dimension, with the controller induced by $P R O C\left(\sharp x_{0}\right)$ for patterns of length 1 . The red line is the target state $x^{o b j}=(0.5,0)$, the black lines are the controlled trajectories. 